\title{
SHORT PRESBURGER ARITHMETIC IS HARD ${ }^{\dagger}$
}

\author{
DANNY NGUYEN* AND IGOR PAK ${ }^{\star}$
}

\begin{abstract}
We study the computational complexity of short sentences in Presburger arithmetic (SHORT-PA). Here by "short" we mean sentences with a bounded number of variables, quantifiers, inequalities and Boolean operations; the input consists only of the integer coefficients involved in the linear inequalities. We prove that satisfiability of SHORT-PA sentences with $m+2$ alternating quantifiers is $\Sigma_{m}^{\mathrm{P}}$-complete or $\Pi_{m}^{\mathrm{P}}$-complete, when the first quantifier is $\exists$ or $\forall$, respectively. Counting versions and restricted systems are also analyzed. Further application are given to hardness of two natural problems in Integer Optimization.
\end{abstract}

\section{INTRODUCTION}

1.1. Outline of the results. We consider short Presburger sentences, defined as follows:

$$
\left(\text { Short-PA } A_{m}\right) \quad \exists \mathbf{x}_{1} \forall \mathbf{x}_{2} \ldots \forall / \exists \mathbf{x}_{m}: \Phi\left(\mathbf{x}_{1}, \ldots, \mathbf{x}_{m}\right) \text {, }
$$

where the quantifiers alternate, the variables $\mathbf{x}_{i} \in \mathbb{Z}^{n_{i}}$ have fixed dimensions $\bar{n}=\left(n_{1}, \ldots, n_{m}\right)$, and $\Phi\left(\mathbf{x}_{1}, \ldots, \mathbf{x}_{m}\right)$ is a fixed Boolean combination of integer linear systems of fixed lengths (numbers of inequalities):

$$
\text { (*) } \quad A_{1} \mathbf{x}_{1}+\ldots+A_{k} \mathbf{x}_{m} \leq \bar{b} .
$$

In other words, everything is fixed in $\left(\right.$ Short-PA $m$ ), except for the entries of the matrices $A_{i}$ and of the vectors $\bar{b}$ in $(*)$. We also call $\Phi$ a short Presburger expression.

The feasibility of short Presburger sentences is a well known open problem which we resolve in this paper. Connected to both Integer Programming and Computational Logic, it was called a "fundamental question" by Barvinok in a recent survey [Bar17]. Many precursors to (Short-PA ${ }_{m}$ ) are well known, including Integer Linear Programming:

$$
\text { (IP) } \exists \mathbf{x}: A \mathbf{x} \leq \bar{b},
$$

and Parametric Integer Programming:

$$
\text { (PIP) } \forall \mathbf{y} \in Q \quad \exists \mathbf{x}: A \mathbf{x}+B \mathbf{y} \leq \bar{b},
$$

where $Q$ is a convex polyhedron given by $K \mathbf{y} \leq \bar{u}$. In both cases, the problems were shown to be in P, by Lenstra in 1982 and Kannan in 1990, respectively (Theorem 1.8). Traditionally, the lengths of the systems in both (IP) and (PIP) are not restricted. However, it is known that they both can be reduced to the case of a bounded length system (c.f. Sec. 8.1 [NP17c] $)$.

\footnotetext{
${ }^{\dagger}$ Extended abstract will appear in Proceedings of the 58th Annual Symposium on Foundations of Computer Science (FOCS 2017).

${ }^{\star}$ Department of Mathematics, UCLA, Los Angeles, CA, 90095. Email: \{ldnguyen, pak\}@math.ucla.edu. October 23, 2017.
} 
Our main result is a complete solution of the problem. We show that for a fixed $m \geq 3$, deciding (Short-PA $\mathrm{P}_{m}$ ) is $\Sigma_{m-2}^{\mathrm{P}}$-complete (Theorem 1.5). This disproves 1 a conjecture by Woods [Woo04, §5.3] (see also [Woo15]), which claims that decision is in P.

Let us emphasize that until this work even the following special case remained open:

$$
\text { (GIP) } \quad \exists \mathbf{z} \in R \quad \forall \mathbf{y} \in Q \exists \mathbf{x}: A \mathbf{x}+B \mathbf{y}+C \mathbf{z} \leq \bar{b},
$$

where $Q$ and $R$ are convex polyhedra given by $K \mathbf{y} \leq \bar{u}$ and $L \mathbf{z} \leq \bar{v}$, respectively. We also show that (GIP) is NP-complete (Theorem [1.2). This resolves an open problem by Kannan Kan92.

Our reduction is parsimonious and also proves that the corresponding counting problem is \#P-complete:

$$
(\# \mathrm{GIP}) \quad \#\{\mathbf{z} \in R: \forall \mathbf{y} \in Q \quad \exists \mathbf{x} \quad A \mathbf{x}+B \mathbf{y}+C \mathbf{z} \leq \bar{b}\} .
$$

There is a natural geometric way to view these problems. Problem (IP) asks whether a given rational polyhedron $P \subset \mathbb{R}^{d}$ contains an integer point. Problem (PIP) asks whether the projection of $P$ contains all integer points in some polyhedron $Q$. Finally, problem (GIP) asks whether there is an $R$-slice of a polyhedron $P$ for which the projection contains all integer points in some polyhedron $Q$.

1.2. Precise statements. For $m=3$ alternating quantifiers, we have the first hard instance of $\left(\right.$ Short-PA $\left.{ }_{m}\right)$ :

$$
\left(\text { Short-PA } \mathrm{PA}_{3}\right) \quad \exists \mathbf{z} \forall \mathbf{y} \exists \mathbf{x}: \Phi(\mathbf{x}, \mathbf{y}, \mathbf{z}) .
$$

Here $\Phi$ is a short Presburger expression in $\mathbf{x}, \mathbf{y}$ and $\mathbf{z}$. We can also define the counting problem

$$
(\# \text { Short-PA } 3) \quad \#\{\mathbf{z}: \forall \mathbf{y} \exists \mathbf{x} \Phi(\mathbf{x}, \mathbf{y}, \mathbf{z})\}
$$

Theorem 1.1. Deciding (Short-PA 3 ) is NP-complete, even for a short Presburger expression $\Phi$ of at most 10 inequalities in 5 variables $z \in \mathbb{Z}, \mathbf{y} \in \mathbb{Z}^{2}, \mathbf{x} \in \mathbb{Z}^{2}$. Similarly, computing (\#Short-PA $\left.{ }_{3}\right)$ in this case is \#P-complete.

For systems of inequalities, we also get:

Theorem 1.2. Deciding (GIP) is NP-complete, even for a system $A \mathbf{x}+B \mathbf{y}+C z \leq \bar{b}$ of at most 24 inequalities in 9 variables $z \in \mathbb{Z}, \mathbf{y} \in \mathbb{Z}^{2}, \mathbf{x} \in \mathbb{Z}^{6}$, when $R$ is an interval and $Q$ is a triangle. Similarly, computing (\#GIP) in this case is \#P-complete.

The third dimension $\mathbf{x} \in \mathbb{Z}^{6}$ in the theorem can be lowered to $\mathbf{x} \in \mathbb{Z}^{3}$ at the cost of increasing the length of the linear system:

Theorem 1.3. Deciding (GIP) is NP-complete, even for a system $A \mathbf{x}+B \mathbf{y}+C z \leq \bar{b}$ of at most 8400 inequalities in 6 variables $z \in \mathbb{Z}, \mathbf{y} \in \mathbb{Z}^{2}, \mathbf{x} \in \mathbb{Z}^{3}$, when $R$ is an interval and $Q$ is a triangle. Similarly, computing (\#GIP) in this case is \#P-complete.

This substantially strengthens our earlier result [NP17c], which considers (GIP) with a "long system", i.e., a system arbitrarily many inequalities:

Theorem 1.4 ([NP17c $)$. Deciding (GIP) is NP-complete, for a system $A \mathbf{x}+B \mathbf{y}+C z \leq \bar{b}$ of unbounded length in 6 variables $z \in \mathbb{Z}, \mathbf{y} \in \mathbb{Z}^{2}, \mathbf{x} \in \mathbb{Z}^{3}$.

\footnotetext{
${ }^{1}$ Assuming the polynomial hierarchy does not collapse.
} 
At the time of proving Theorem 1.4, we thought it would be the strongest negative result (see Section 1.5 below). Nevertheless, the new results in theorems 1.1, 1.2 and 1.3 say that at the level of three quantifiers, both Integer Programming and Presburger Arithmetic quickly saturate to a high level of complexity, even when all parameters are bounded.

The decision part of Theorem 1.1 can naturally be generalized to short Presburger sentences of more than 3 quantifiers:

Theorem 1.5 (Main result). Fix $m \geq 1$. Let $Q_{1}, \ldots, Q_{m+2} \in\{\forall, \exists\}$ be $m+2$ alternating quantifiers with $Q_{1}=\exists$. Deciding short Presburger sentences of the form

$$
\begin{array}{lllll}
Q_{1} \mathbf{z}_{1} & \ldots & Q_{m+1} \mathbf{z}_{m+1} & Q_{m+2} \mathbf{z}_{m+2} & :
\end{array}\left(\mathbf{z}_{1}, \ldots, \mathbf{z}_{m+2}\right)
$$

is $\Sigma_{m}^{\mathrm{P}}$-complete. Similarly, when $Q_{1}=\forall$, deciding short Presburger sentences as above is $\Pi_{m}^{\mathrm{P}}$-complete. Here $\Phi$ is a short Presburger expression of at most $10 \mathrm{~m}$ inequalities in $4 m+1$ variables $\mathbf{z}_{1} \in \mathbb{Z}, \mathbf{z}_{2}, \mathbf{z}_{m+2} \in \mathbb{Z}^{2}$, and $\mathbf{z}_{3}, \ldots, \mathbf{z}_{m+1} \in \mathbb{Z}^{4}$.

The proof of the above results uses a chain of reductions. We start with the AP-COVER problem on covering intervals with arithmetic progressions. This problem is NP-compete by a result of Stockmeyer and Meyer [SM73] (see Section 9). The arithmetic progressions are encoded via continued fractions by a single rational number $p / q$. We use the plane geometry of continued fractions and "lift" the construction to a Boolean combination of polyhedra in dimension 5, proving Theorem 1.1. We then "lift" the construction further to convex polytopes $Q_{1} \subset \mathbb{R}^{9}$ and $Q_{2} \subset \mathbb{R}^{6}$, which give proofs of theorems 1.2 and 1.3, respectively. While both constructions are explicit, the first construction gives a description of $Q_{1}$ by its 24 facets, while the second gives a description of $Q_{2}$ by its 40 vertices; the bound of 8400 facets then comes from McMullen's Upper bound theorem (Theorem 5.1). Finally, we generalize the problem AP-COVER and the chain of reductions to $m \geq 3$ quantifiers.

1.3. Applications in integer optimization. The first application of our construction is the following hardness result on the bilevel optimization of a quadratic function over integer points in a polytope.

Theorem 1.6. Given a rational interval $J \subset \mathbb{R}$, a rational polytope $W \subset \mathbb{R}^{5}$ and a quadratic rational polynomial $h: \mathbb{R}^{6} \rightarrow \mathbb{R}$, computing:

$$
\max _{z \in J \cap \mathbb{Z}} \min _{\mathbf{w} \in W \cap \mathbb{Z}^{5}} h(z, \mathbf{w})
$$

is NP-hard. This holds even when $W$ has at most 18 facets.

The polytope $W$ can be given either by its vertices or by its facets, as the theorem holds in both cases.

The second application is to the hardness of the Pareto optima. Assume we are given polytope $Q \subset \mathbb{R}^{n}$, and $k$ functions $f_{1}, \ldots, f_{k}: \mathbb{R}^{n} \rightarrow \mathbb{R}$ restricted to the domain $Q \cap \mathbb{Z}^{n}$. For a point $\mathbf{x} \in Q \cap \mathbb{Z}^{n}$, the corresponding outcome vector $\mathbf{y}=\left(f_{1}(\mathbf{x}), \ldots, f_{k}(\mathbf{x})\right)$ is called a Pareto minimum, if there is no other point $\widetilde{\mathbf{x}} \in Q \cap \mathbb{Z}^{n}$ and $\widetilde{\mathbf{y}}=\left(f_{1}(\widetilde{\mathbf{x}}), \ldots, f_{k}(\widetilde{\mathbf{x}})\right)$, such that $\widetilde{\mathbf{y}} \leq \mathbf{y}$ coordinate-wise and $\widetilde{\mathbf{y}} \neq \mathbf{y}$. The goal is to minimize the value of an objective function $g: \mathbb{R}^{k} \rightarrow \mathbb{R}$ over all Pareto minima $\mathbf{y}$ of $\left(f_{1}, \ldots, f_{k}\right)$ on $Q$.

Theorem 1.7. Given a rational polytope $Q \subset \mathbb{R}^{6}$, two rational linear functions $f_{1}, f_{2}$ : $\mathbb{R}^{6} \rightarrow \mathbb{R}$, a rational quadratic polynomial $f_{3}: \mathbb{R}^{6} \rightarrow \mathbb{R}$, and rational linear objective function $g: \mathbb{R}^{3} \rightarrow \mathbb{R}$, computing the minimum of $g$ over the Pareto minima of $\left(f_{1}, f_{2}, f_{3}\right)$ on $Q$ is NP-hard. Moreover, the corresponding 1/2-approximation problem is also NP-hard. This holds even when $Q$ has at most 38 facets. 
Again, the polytope $Q$ can be given either by its vertices or by its facets. Here by $\varepsilon$-approximation we mean approximation up to a multiplicative factor of $\varepsilon$.

We prove both theorems in Section 8 , See also $\$ 11.6$ and $\$ 11.7$ for some background and open problems.

1.4. Historical overview. Presburger Arithmetic was introduced by Presburger in [Pre29], where he proved it is a decidable theory. The general theory allows unbounded numbers of quantifiers, variables and Boolean operations. A quantifier elimination (deterministic) algorithm was given by Cooper [Coo72], and was shown to be triply exponential by Oppen Opp78 (see also [RL78]). A nondeterministic doubly exponential complexity lower bound was obtained by Fischer and Rabin [FR74] for the general theory. This pioneering result was further refined to a triply exponential deterministic lower bound (with unary output) in [Wei97, and a simply exponential nondeterministic lower bound for a bounded number of quantifier alternations [Für82] (see also [Sca84]). Of course, in all these cases the number of variables is unbounded.

In Sch97, Schöning proved NP-completeness for two quantifiers $\exists y \forall x: \Phi(x, y)$, where $x, y \in \mathbb{Z}$ and $\Phi(x, y)$ is a Presburger expression in 2 variables, i.e., a Boolean combination of arbitrarily many inequalities in $x, y$. This improved on an earlier result by Grädel, who also established that similar sentences with $m+1$ alternating quantifiers and a bounded number of variables are complete for the $m$-th level in the Polynomial Hierarchy [Grä87. Roughly speaking, one can view our results as variations on Grädel's result, where we trade boundedness of $\Phi$ for an extra quantifier.

Let us emphasize that when the number of variables is unbounded, even the most simple systems (IP) become NP-complete. The examples include the KNAPSACK, one of the oldest NP-complete problems [GJ79]. Note also that even when matrix $A$ has at most two nonzero entries in each row, the problem remains NP-complete Lag85.

In a positive direction, the progress has been limited. The first breakthrough was made by Lenstra Len83] (see also [Sch86]), who showed that (IP) can be solved in polynomial time in a fixed dimension (see also [Eis03 for better bounds). Combined with a reduction by Scarpellini [Sca84], this implies that deciding (Short-PA $\left.{ }_{1}\right)$ is in $\mathrm{P}$.

The next breakthrough was made by Kannan [Kan90] (see also Kan92]), who showed that (PIP) in fixed dimensions is in $\mathrm{P}$, even if the number $s$ of inequalities is unbounded, i.e. the matrices $A$ and $B$ can be "long". This was a motivation for our earlier Theorem 1.4 from [NP17c], which ruled out "long" systems for (GIP).

Theorem 1.8 (Kannan). Fix $n_{1}, n_{2}$. The formula (PIP) in variables $\mathbf{x} \in \mathbb{Z}^{n_{1}}, \mathbf{y} \in \mathbb{Z}^{n_{2}}$ with $s$ inequalities can be decided in polynomial time, where $s$ is part of the input.

Kannan's Theorem was further strengthened by Eisenbrand and Shmonin [ES08] (see \$10.2). All of these greatly contrast with the above hardness results by Schöning and Grädel, because here only conjunctions of inequalities are allowed.

The corresponding counting problems have also been studied with great success. First, Barvinok Bar93] showed that integer points in a convex polytope $P \subset \mathbb{R}^{d}$ can be counted in polynomial time, for a fixed dimension $n$ (see also [Bar06, BP99]). He utilized the short generating function approach pioneered by Brion, Vergne and others (see [Bar08] for details and references). Woods Woo04] extended this approach to general Boolean formulas.

In the next breakthrough, Barvinok and Woods showed how to count projections of integer points in a (single) polytope in polynomial time BW03. Woods Woo04 extended this approach to general Presburger expressions $\Phi$ with a fixed number of inequalities (see 
also Woo15 and an alternative proof in NP17a]). As a consequence, he showed that deciding $\left(\right.$ Short- $\mathrm{PA}_{2}$ ) is in $\mathrm{P}$. This represents the most general positive result in this direction:

Theorem 1.9 (Woods). Fix $n_{1}, n_{2}$ and s. Given a short Presburger expression $\Phi(\mathbf{x}, \mathbf{y})$ in variables $\mathbf{x} \in \mathbb{Z}^{n_{1}}, \mathbf{y} \in \mathbb{Z}^{n_{2}}$ with at most $s$ inequalities, the sentence

$$
\forall \mathbf{y} \exists \mathbf{x}: \Phi(\mathbf{x}, \mathbf{y})
$$

can be decided in polynomial time. Moreover, the number of solutions

$$
\#\{\mathbf{y}: \exists \mathbf{x} \Phi(\mathbf{x}, \mathbf{y})\}
$$

can be computed in polynomial time.

1.5. Kannan's Partition Theorem. In Kan90, Kannan introduced the technology of test sets for efficient solutions of (PIP). The Kannan Partition Theorem (KPT), see Theorem 10.1 below, claims that one can find in polynomial time a partition of the $k$-dimensional parameter space $W$ into polynomially many rational (co-)polyhedra

$$
\text { (o) } \quad W=P_{1} \sqcup P_{2} \sqcup \ldots \sqcup P_{r} \text {, }
$$

so that only a bounded number of tests need to be performed (see $₫ 10.1$ for precise statement details).

In NP17a, we showed that KPT if valid would imply a polynomial time decision algorithm for $($ Short-PA $m$ ), and in particular (GIP) for a restricted system. Thus, at the time of proving Theorem 1.4 in [NP17c], we thought that [NP17a] and [NP17c] together would completely characterize the complexity of (GIP), depending on whether the system is restricted or not.

In view of our theorems 1.1, 1.2, 1.3 and 1.5, it strongly suggests that KPT may actually be erroneous. However, we did not expect this at the time of writing [NP17a. In fact, the prevailing view was that $($ Short-PA $m$ ) would always be in $\mathrm{P}$, which neatly aligned with the results in [NP17a] (conditional upon KPT). Now that the hardness results are known, we are actually able combine the current techniques with some of those in [NP17a] to obtain the following quantitative result, which strongly contradicts KPT:

Theorem 1.10. Fix $m, n$ and let $k=1$. Let $\phi$ be the total bit length of the matrix $A \in$ $\mathbb{Z}^{m \times n}$ in KPT. Then for the number $r$ of pieces in Kannan's partition (o), we must have $r>\exp (\varepsilon \phi)$ for some constant $\varepsilon=\varepsilon(n, m)>0$.

We conclude no polynomial size partition (o) exists as claimed by KPT. See Section 10 for a detailed presentation of this result and its implications, 11.1 for our point of view, and $§ 11.2$ for the gap in the original proof of KPT.

\section{Notations}

We use $\mathbb{N}=\{0,1,2, \ldots\}$ and $\mathbb{Z}_{+}=\{1,2,3, \ldots\}$

Universal/existential quantifiers are denoted $\forall / \exists$.

Unspecified quantifiers are denoted by $Q_{1}, Q_{2}$, etc.

Unquantified Presburger expressions are denoted by $\Phi, \Psi$, etc.

We use $\left[\begin{array}{l}a \\ b\end{array}\right]$ for a disjunction $(a \vee b)$ and $\left\{\begin{array}{l}a \\ b\end{array}\right\}$ for a conjunction $(a \wedge b)$.

All constant vectors are denoted $\bar{n}, \bar{b}, \bar{\alpha}, \bar{\nu}$, etc.

We use 0 to denote both zero and the zero vector.

All matrices are denoted $A, B, C$, etc.

All integer variables are denoted $x, y, z$, etc. 
All vectors of integer variables are denoted $\mathbf{x}, \mathbf{y}, \mathbf{z}$, etc.

In a vector $\mathbf{y}=\left(y_{1}, y_{2}\right)$, we draw $y_{2}$ as a vertical and $y_{1}$ as a horizontal coordinate.

We use $\lfloor$.$\rfloor to denote the floor function.$

The the vector $\mathbf{y}$ with coordinates $y_{i}=\left\lfloor x_{i}\right\rfloor$ is denoted by $\mathbf{y}=\lfloor\mathbf{x}\rfloor$.

Half-open intervals are denoted by $[\alpha, \beta),(\alpha, \beta]$, etc.

A polyhedron is an intersection of finitely many closed half-spaces in $\mathbb{R}^{n}$.

A copolyhedron is a polyhedron with possibly some open facets.

A polytope is a bounded polyhedron.

Subsets of $\mathbb{N}$ are denoted by $\Gamma, \Delta$, etc.

\section{BASIC PROPERTIES OF FINITE CONTINUED FRACTIONS}

Every rational number $\alpha>1$ can be written in the form:

$$
\alpha=\left[a_{0} ; a_{1}, \ldots, a_{n}\right]=a_{0}+\frac{1}{a_{1}+\frac{1}{\ddots \cdot+\frac{1}{a_{n}}}},
$$

where $a_{0}, \ldots, a_{n} \in \mathbb{Z}_{+}$. If $a_{n}>1$, we have another representation:

$$
\alpha=\left[a_{0} ; a_{1}, \ldots, a_{n}-1,1\right]=a_{0}+\frac{1}{a_{1}+\frac{1}{\ddots \cdot+\frac{1}{\left(a_{n}-1\right)+\frac{1}{1}}}} .
$$

On the other hand, if $a_{n}=1$, then we also have:

$$
\alpha=\left[a_{0} ; a_{1}, \ldots, a_{n-1}, 1\right]=\left[a_{0} ; a_{1}, \ldots, a_{n-1}+1\right] .
$$

It is well known that any rational $\alpha>1$ can be written as a continued fraction as above in exactly two ways (see e.g. [Kar13, Khi64]), one with an odd number of terms and the other one with an even number of terms.

If a continued fraction $\left[a_{0} ; a_{1}, \ldots, a_{n}\right]$ evaluates to a rational value $p / q$, we identify it with the integer point $(q, p)$. We write:

$$
(q, p) \leftrightarrow\left[a_{0} ; a_{1}, \ldots, a_{n}\right] .
$$

From now on, we will only consider continued fractions with an odd number of terms:

$$
\alpha=\left[a_{0} ; a_{1}, \ldots, a_{2 k}\right] .
$$

To facilitate later computations, we will relabel these $2 k+1$ terms as:

$$
\alpha=\left[a_{0} ; b_{0}, a_{1}, b_{1}, \ldots, a_{k-1}, b_{k-1}, a_{k}\right] .
$$

The convergents of $\alpha$ are 2-dimensional integer vectors, defined as:

$$
\begin{gathered}
C_{0}=(1,0), D_{0}=(0,1), \\
C_{i}=a_{i-1} D_{i-1}+C_{i-1}, \text { for } i=1, \ldots, k+1, \\
D_{i}=b_{i-1} C_{i}+D_{i-1}, \text { for } i=1, \ldots, k .
\end{gathered}
$$

We call $C_{0}, D_{0}, \ldots, C_{k}, D_{k}, C_{k+1}$ the convergents for $\alpha$. If $C_{i}=\left(q_{i}, p_{i}\right)$ and $D_{i}=\left(s_{i}, r_{i}\right)$ then we have the properties: 
P1) $p_{0}=0, q_{0}=1, r_{0}=1, s_{0}=0$.

P2) $p_{i}=a_{i-1} r_{i-1}+p_{i-1}, q_{i}=a_{i-1} s_{i-1}+q_{i-1}$.

P3) $r_{i}=b_{i-1} p_{i}+r_{i-1}, s_{i}=b_{i-1} q_{i}+s_{i-1}$.

P4) $C_{i+1}=\left(q_{i+1}, p_{i+1}\right) \leftrightarrow\left[a_{0} ; b_{0}, a_{1}, b_{1}, \ldots, b_{i-1}, a_{i}\right]$.

P5) The quotients $p_{i} / q_{i}$ form an increasing sequence, starting with $p_{0} / q_{0}=0$ and ending with $p_{k+1} / q_{k+1}=\alpha$.

P6) $D_{i+1}=\left(s_{i+1}, r_{i+1}\right) \leftrightarrow\left[a_{0} ; b_{0}, a_{1}, b_{1}, \ldots, a_{i}, b_{i}\right]$.

P7) The quotients $r_{i} / s_{i}$ form a decreasing sequence, starting with $r_{0} / s_{0}=\infty$, and ending with $r_{k} / s_{k}=\left[a_{0} ; b_{0}, a_{1}, b_{1}, \ldots, a_{k-1}, b_{k-1}\right]$.

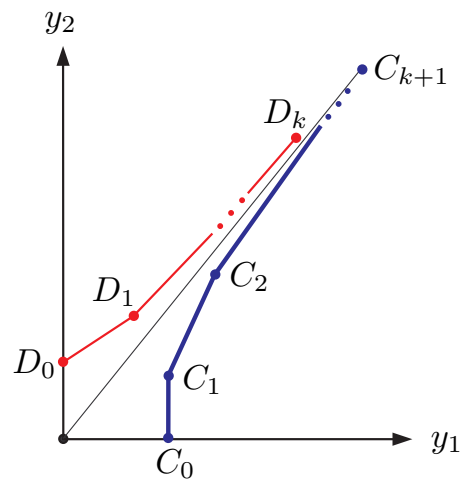

Figure 1. The curves $\mathcal{C}$ (bold) and $\mathcal{D}$.

Denote by $O$ the origin in $\mathbb{Z}^{2}$. The geometric properties of these convergents are:

G1) Each vector $\overrightarrow{O C_{i}}$ and $\overrightarrow{O D_{i}}$ is primitive in $\mathbb{Z}^{2}, \operatorname{meaning} \operatorname{gcd}\left(p_{i}, q_{i}\right)=\operatorname{gcd}\left(r_{i}, s_{i}\right)=1$.

G2) Each segment $C_{i} C_{i+1}$ contains exactly $a_{i}+1$ integer points, since $\overrightarrow{C_{i} C_{i+1}}=a_{i} \overrightarrow{O D_{i}}$.

G3) Each segment $D_{i} D_{i+1}$ contains exactly $b_{i}+1$ integer points, since $\overrightarrow{D_{i} D_{i+1}}=b_{i} \overrightarrow{O C_{i+1}}$.

G4) The curve $\mathcal{C}$ connecting $C_{0}, C_{1}, \ldots, C_{k+1}$ is (strictly) convex upward (see Figure 1).

G5) The curve $\mathcal{D}$ connecting $D_{0}, D_{1}, \ldots, D_{k}$ is (strictly) convex downward.

G6) There are no interior integer points above $\mathcal{C}$ and below $\overrightarrow{O C_{k+1}}$. In other words, $\mathcal{C}$ is the upper envelope of all non-zero integer points between $\overrightarrow{O C_{0}}$ and $\overrightarrow{O C_{k+1}}$.

\section{From arithmetic progressions to short Presburger sentences}

4.1. Covering with arithmetic progressions. For a triple $(g, h, e) \in \mathbb{N}^{3}$, denote by $\operatorname{AP}(g, h, e)$ the arithmetic progression:

$$
\operatorname{AP}(g, h, e)=\{g+j e: 0 \leq j \leq h\} .
$$

We reduce the following classical NP-complete problem to (Short-PA 3 ):

\section{AP-COVER}

Input: $\quad$ An interval $J=[\mu, \nu] \subset \mathbb{Z}$ and $k$ triples $\left(g_{i}, h_{i}, e_{i}\right)$ for $i=1, \ldots, k$.

Decide: Is there $z \in J$ such that $z \notin \mathrm{AP}_{1} \cup \cdots \cup \mathrm{AP}_{k}$, where $\operatorname{AP}_{i}=\operatorname{AP}\left(g_{i}, h_{i}, e_{i}\right)$ ?

The problem AP-COVER was shown to be NP-complete by Stockmeyer and Meyer (Theorem 9.1). A short proof of this is included in 99.1 for completeness. We remark that the inputs $\mu, \nu, g_{i}, h_{i}, e_{i}$ to the problem are in binary. We can assume that each $h_{i} \geq 1$, i.e., 
each $\mathrm{AP}_{i}$ contains more than 1 integer. This is because we can always increase $\nu \leftarrow \nu+1$ and add the last integer $\nu+1$ to any progression $\mathrm{AP}_{i}$ that previously had only a single element. Note that AP-COVER is also invariant under translation, so we can assume that $\mu, \nu$ and all $g_{i}, h_{i}, e_{i}$ are positive integers.

Next, let:

$$
M=1+\nu \prod_{i=1}^{k} g_{i}\left(g_{i}+h_{i} e_{i}\right)
$$

We have:

$$
M>\nu \quad \text { and } \quad M>\max _{i}\left(g_{i}+h_{i} e_{i}\right) .
$$

i.e., the interval $[1, M-1]$ contains $J$ and all $\mathrm{AP}_{i}$. Moreover, we have:

$$
\operatorname{gcd}\left(M, g_{i}\right)=\operatorname{gcd}\left(M, g_{i}+h_{i} e_{i}\right)=1, i=1, \ldots, k .
$$

Note that $M$ can be computed in polynomial time from the input of AP-COVER, and

$$
\log M=O\left(\sum_{i=1}^{k} \log g_{i}+\log h_{i}+\log e_{i}\right) .
$$

Let us construct a continued fraction

$$
\alpha=\left[a_{0} ; b_{0}, a_{1}, b_{1}, \ldots, a_{2 k-2}, b_{2 k-2}, a_{2 k-1}\right]
$$

with the following properties:

1) All $a_{i}, b_{j} \in[1, M]$.

2) For each $1 \leq i<k$, we have $a_{2 i}=1$.

3) For each $1 \leq i \leq k$, we have $a_{2 i-1}=h_{i}$.

4) For each $1 \leq i \leq k$, if

$$
C_{2 i-1}:=\left(q_{2 i-1}, p_{2 i-1}\right) \leftrightarrow\left[a_{0} ; b_{0}, \ldots, a_{2 i-2}\right]
$$

then we have $p_{2 i-1} \equiv g_{i}(\bmod M)$.

5) For each $1 \leq i \leq k$, if

$$
C_{2 i}:=\left(q_{2 i}, p_{2 i}\right) \leftrightarrow\left[a_{0} ; b_{0}, \ldots, a_{2 i-1}\right]
$$

then we have $p_{2 i} \equiv g_{i}+h_{i} e_{i}(\bmod M)$.

6) For each $1 \leq i \leq k$, the segment $C_{2 i-1} C_{2 i}$ contains exactly $h_{i}+1$ integer points. Moreover, the set

$$
\mathcal{A}_{i}:=\left\{y_{2} \bmod M:\left(y_{1}, y_{2}\right) \in C_{2 i-1} C_{2 i}\right\}
$$

is exactly $\mathrm{AP}_{i}$.

7) For each $1 \leq i<k$, the segment $C_{2 i} C_{2 i+1}$ contains no integer points apart from the two end points.

We construct $\alpha$ iteratively as follows. We say an integer vector $Y=\left(y_{1}, y_{2}\right)$ is congruent to $z \bmod M$, denoted $Y \equiv z(\bmod M)$, if $y_{2} \equiv z(\bmod M)$. As in (3.1), let $C_{0}=(1,0)$ and $D_{0}=(0,1)$.

Step 1: Let $a_{0}=g_{1}$. Then

$$
C_{1}=a_{0} D_{0}+C_{0}=\left(1, g_{1}\right) \text { and } C_{1} \equiv g_{1}(\bmod M) .
$$


Step 2: Take $b_{0}$ so that

$$
D_{1}=b_{0} C_{1}+D_{0}=\left(b_{0}, b_{0} g_{1}\right)+(0,1) \equiv e_{1}(\bmod M),
$$

i.e.,

$$
b_{0} g_{1}+1 \equiv e_{1}(\bmod M) .
$$

We can solve for $b_{0} \bmod M$ because $\operatorname{gcd}\left(M, g_{1}\right)=1$ from (4.1). So there exists $b_{0} \in[1, M]$ s.t. $D_{1} \equiv e_{1}(\bmod M)$.

Step 3: Take $a_{1}=h_{1}$. This implies

$$
C_{2}=a_{1} D_{1}+C_{1} \equiv h_{1} e_{1}+g_{1}(\bmod M) .
$$

By Property (G2), we also have exactly $h_{1}+1$ integer points on $C_{1} C_{2}$.

Observation: After these steps, we have $h_{1}+1$ integer points on $C_{1} C_{2}$. Every two such consecutive points differ by $\overrightarrow{O D_{1}}$. Reduced $\bmod M$, they give:

$$
C_{1} \equiv g_{1}, g_{1}+e_{1}, \ldots, g_{1}+h_{1} e_{1} \equiv C_{2}(\bmod M) .
$$

Thus, we have $\mathcal{A}_{1}=\mathrm{AP}_{1}$. Conditions (1)-(7) hold so far.

Step 4: Take $b_{1}$ so that $D_{2} \equiv g_{2}-\left(g_{1}+h_{1} e_{1}\right)(\bmod M)$. Since we have the recurrence

$$
D_{2}=b_{1} C_{2}+D_{1} \equiv b_{1}\left(g_{1}+h_{1} e_{1}\right)+e_{1}(\bmod M)
$$

this is equivalent to solving

$$
b_{1}\left(g_{1}+h_{1} e_{1}\right)+e_{1} \equiv g_{2}-\left(g_{1}+h_{1} e_{1}\right)(\bmod M) .
$$

Again we can solve for $b_{1} \bmod M$ because $\operatorname{gcd}\left(M, g_{1}+h_{1} e_{1}\right)=1$ from (4.1). So there exists $b_{1} \in[1, M]$ s.t. $D_{2} \equiv g_{2}-\left(g_{1}+h_{1} e_{1}\right)(\bmod M)$.

Step 5: Take $a_{2}=1$. This implies

$$
\begin{aligned}
C_{3}=a_{2} D_{2}+C_{2} & \equiv g_{2}-\left(g_{1}+h_{1} e_{1}\right)+g_{1}+h_{1} e_{1} \\
& \equiv g_{2}(\bmod M) .
\end{aligned}
$$

This satisfies condition (4) for $i=2$. Now we can start encoding $\mathrm{AP}_{2}$ with $C_{3}(\bmod M)$.

Observation: One can see that $b_{1}$ in Step 4 was appropriately set up to facilitate Step 5. It is conceptually easier to start with Step 5 and retrace to get the appropriate condition for $b_{1}$. Taking $a_{2}=1$ also implies that there are no other integer points on $C_{2} C_{3}$ apart from the two endpoints.

Step 6: Take $b_{2}$ so that $D_{3}=b_{2} C_{3}+D_{2} \equiv e_{2}(\bmod M)$. This is similar to Step 2 . Again we use condition (4.1).

Step 7: Take $a_{3}=h_{2}$, which implies

$$
C_{4}=a_{3} D_{3}+C_{3} \equiv g_{2}+h_{2} e_{2}(\bmod M) .
$$

After this, we again get exactly $h_{2}+1$ integer points on $C_{3} C_{4}$. Reduced mod $M$, they give $\mathcal{A}_{2}=\mathrm{AP}_{2}$. Note that conditions (1)-(7) still hold.

The rest proceeds similarly to Steps $4-7$, for $2 \leq j \leq k-1$ :

Step $4 j$ : Take $b_{2 j-1}$ so that

$$
D_{2 j} \equiv g_{j+1}-\left(g_{j}+h_{j} e_{j}\right)(\bmod M) .
$$


Step $4 j+1$ : Take $a_{2 j}=1$, which implies

$$
C_{2 j+1}=D_{2 j}+C_{2 j} \equiv g_{j+1}(\bmod M) .
$$

Step $4 j+2$ : Take $b_{2 j}$ so that $D_{2 j+1} \equiv e_{j+1}(\bmod M)$.

Step $4 j+3$ : Take $a_{2 j+1}=h_{j+1}$, which implies

$$
C_{2 j+2} \equiv g_{j+1}+h_{j+1} e_{j+1}(\bmod M) \text {. }
$$

The segment $C_{2 j+1} C_{2 j+2}$ contains exactly $h_{j+1}+1$ integer points.

Observation: After these four steps, we get $\mathcal{A}_{j+1}=\mathrm{AP}_{j+1}$. Conditions (1)-(7) hold throughout.

All modular arithmetic mod $M$ in the above procedure can be performed in polynomial time. The last Step $4 k-1$ gives:

$$
C_{2 k}=\left(q_{2 k}, p_{2 k}\right) \leftrightarrow\left[a_{0} ; b_{0}, a_{1}, b_{1}, \ldots, a_{2 k-1}\right] .
$$

All terms $a_{i}$ and $b_{j}$ are in the range $[1, M]$, so the final quotient $p_{2 k} / q_{2 k}$ can be computed in polynomial time using the recurrence (3.1). This implies that $p_{2 k}$ and $q_{2 k}$ have polynomial binary lengths compared to the input $\mu, \nu, g_{i}, h_{i}, e_{i}$ of AP-COVER. The curve $\mathcal{C}$ connecting $C_{0}, C_{1}, \ldots, C_{2 k}$ is shown in Figure 2 .

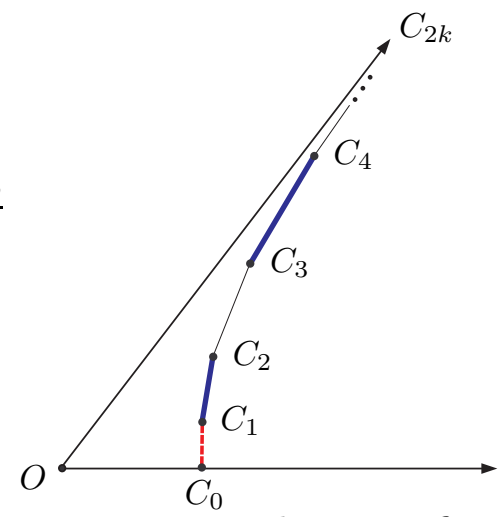

Figure 2. The curve $\mathcal{C}$.

Here each bold segment $C_{2 i-1} C_{2 i}$ contains $h_{i}+1$ integer points. Each thin black segment $C_{2 i} C_{2 i+1}$ contains no interior integer points. The dotted segment $C_{0} C_{1}$ contains $g_{1}+1$ integer points, the first $g_{1}$ of which we will not need. Let $\mathcal{C}^{\prime}$ be $\mathcal{C}$ minus the first $g_{1}$ integer points on $C_{0} C_{1}$. For brevity, we also denote $C_{2 k}=\left(q_{2 k}, p_{2 k}\right)=(q, p)$.

4.2. Analysis of the construction. We define:

$$
\Delta=\left\{z: \exists\left(y_{1}, y_{2}\right) \in \mathcal{C}^{\prime} \quad z \equiv y_{2}(\bmod M)\right\} .
$$

By condition (7), every integer point $\mathbf{y}=\left(y_{1}, y_{2}\right) \in \mathcal{C}^{\prime}$ lies on one of the segments $C_{1} C_{2}$, $C_{3} C_{4}, \ldots, C_{2 k-1} C_{2 k}$. Moreover, by condition (6), for $1 \leq i \leq k$ we have:

$$
\mathrm{AP}_{i}=\mathcal{A}_{i}=\left\{z: \exists \mathbf{y} \in C_{2 i-1} C_{2 i} z \equiv y_{2}(\bmod M)\right\}
$$

Therefore, we have:

$$
\mathrm{AP}_{1} \cup \cdots \cup \mathrm{AP}_{k}=\mathcal{A}_{1} \cup \cdots \cup \mathcal{A}_{k}=\Delta .
$$

Recall that AP-COVER asks whether:

$$
\exists z \in J \quad z \notin \mathrm{AP}_{1} \cup \cdots \cup \mathrm{AP}_{k} \Longleftrightarrow \exists z \in J \quad z \notin \Delta .
$$


By (4.2), this is equivalent to:

$$
\exists z \in J \quad \forall \mathbf{y} \in \mathcal{C}^{\prime} \quad z \not \equiv y_{2}(\bmod M),
$$

which can be rewritten as:

$$
\exists z \in J \quad \forall \mathbf{y} \quad z \not \equiv y_{2}(\bmod M) \vee \mathbf{y} \notin \mathcal{C}^{\prime} .
$$

Next, we express the condition $\mathbf{y}=\left(y_{1}, y_{2}\right) \in \mathcal{C}^{\prime}$ in short Presburger arithmetic. Let $\mathbf{v}=(p,-q)$ and $\theta$ be the cone between $\overrightarrow{O C_{0}}$ and $\overrightarrow{O C_{2 k}}$, i.e.,

$$
\theta=\left\{\mathbf{y} \in \mathbb{R}^{2}: y_{2} \geq 0, \mathbf{v} \cdot \mathbf{y} \geq 0\right\} .
$$

For each $\mathbf{y}=\left(y_{1}, y_{2}\right) \in \theta$, denote by $P_{\mathbf{y}}$ the parallelogram with two opposite vertices $O$ and y and sides parallel to $\overrightarrow{O C_{0}}$ and $\overrightarrow{O C_{2 k}}$ (see Figure 3 ). We also require that horizontal edges in $P_{\mathbf{y}}$ are open, i.e.,

$$
P_{\mathbf{y}}=\left\{\mathbf{x} \in \mathbb{R}^{2}: \begin{array}{r}
\mathbf{v} \cdot \mathbf{y} \geq \mathbf{v} \cdot \mathbf{x} \geq 0 \\
y_{2}>x_{2}>0
\end{array}\right\}
$$

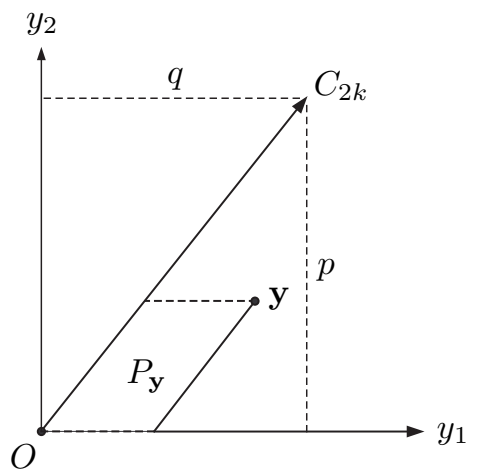

Figure 3. The parallelogram $P_{\mathbf{y}}$. The upper and lower edges of $P_{\mathbf{y}}$ are open (dotted). Here we denote $C_{2 k}=\left(q_{2 k}, p_{2 k}\right)=(q, p)$.

Lemma 4.1. For $\mathbf{y} \in \mathbb{Z}^{2}$, we have:

$$
\mathbf{y} \in \mathcal{C}^{\prime} \Longleftrightarrow \mathbf{v} \cdot \mathbf{y} \geq 0 \wedge y_{2} \geq g_{1} \wedge P_{\mathbf{y}} \cap \mathbb{Z}^{2}=\varnothing .
$$

Proof. First, assume $\mathbf{y}:=\left(y_{1}, y_{2}\right) \in \mathcal{C}^{\prime}$. Recall that $\mathcal{C}^{\prime}$ is $\mathcal{C}$ minus the first $g_{1}$ integer points on $C_{0} C_{1}$. Therefore, we have $y_{2} \geq g_{1}$. Since $\mathcal{C}$ sits inside $\theta$, we also have $\mathbf{y} \in \theta$, which implies $\mathbf{v} \cdot \mathbf{y} \geq 0$. Let $\mathcal{R}$ be the concave region above $\mathcal{C}$ and below $\overrightarrow{O C_{2 k}}$. By property (G6), $\mathcal{R}$ contains no interior integer points. Since $\mathbf{y} \in \mathcal{C}$, we have $P_{\mathbf{y}} \subset \mathcal{R}$. Therefore, the parallelogram $P_{\mathbf{y}}$ in (4.4) contains no integer points. We conclude that $\mathbf{y}$ satisfies the RHS in (4.5).

Conversely, assume $\mathbf{y}$ satisfies the RHS in (4.5) but $\mathbf{y} \notin \mathcal{C}^{\prime}$. The following argument is illustrated in Figure 4. First, $\mathbf{v} \cdot \mathbf{y} \geq 0 \wedge y_{2} \geq g_{1}$ implies $\mathbf{y} \in \theta$. Also, the parallelogram $P_{\mathbf{y}}$ contains no integer points. By property $(\mathrm{G} 6)$, if $\mathbf{y} \notin \mathcal{C}^{\prime}$, it must lie strictly below $\mathcal{C}^{\prime}$. Let $\mathbf{x}$ and $\mathbf{x}^{\prime}$ be the integer points on $\mathcal{C}$ that are immediately above and below $\mathbf{y}$ (see Figure 4). In other words, $\mathbf{x} \in \mathcal{C}$ is the integer point immediately above the intersection of $\mathcal{C}$ with the upper edge of $P_{\mathbf{y}}$, and $\mathbf{x}^{\prime} \in \mathcal{C}$ is the integer point immediately below the intersection of $\mathcal{C}$ with the right edge of $P_{\mathbf{y}}$. Since $P_{\mathbf{y}}$ contains no integer points, particularly those on $\mathcal{C}$, 
the points $\mathbf{x}$ and $\mathbf{x}^{\prime}$ must be adjacent on $\mathcal{C}$, i.e., they form a segment on $\mathcal{C} \mathbb{2}^{2}$ Now we draw a parallelogram $D$ with two opposite vertices $\mathbf{x}, \mathbf{x}^{\prime}$ and edges parallel to those of $P_{\mathbf{y}}$ (the dashed bold parallelogram in Figure 44). It is clear that $D$ lies inside $\theta$ and also contains $\mathbf{y}$. Take $\mathbf{y}^{\prime}$ to be the reflection of $\mathbf{y}$ across the midpoint of $\mathbf{x x}^{\prime}$. Since $\mathbf{x}, \mathbf{x}^{\prime}$ and $\mathbf{y}$ are integer points, so is $\mathbf{y}^{\prime}$. We also have $\mathbf{y}^{\prime} \in D \subset \theta$. Note also that $\mathbf{y}^{\prime}$ lies on the opposite side of $\mathcal{C}$ compared to $\mathbf{y}$. Therefore, we have $\mathbf{y}^{\prime} \in \mathcal{R}$, contradicting property $(G 6)$.

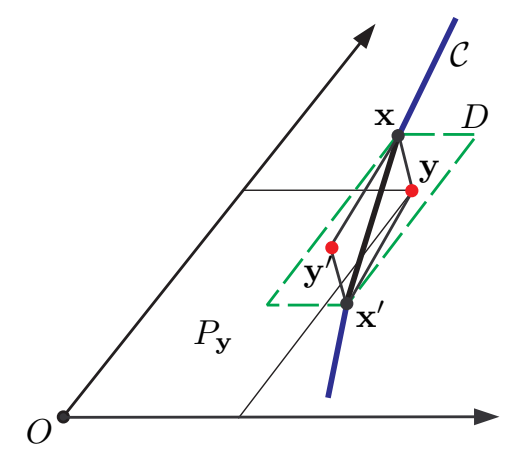

FiguRE 4. $\mathbf{y}^{\prime}$ is the reflection of $\mathbf{y}$ across the midpoint of $\mathbf{x x}^{\prime}$.

Remark 4.2. There is a subtle point about the existence of $\mathrm{x}^{\prime}$ in the above proof. It is clear that $\mathbf{x}$ exists because $\mathbf{y}$ lies below $\mathcal{C}$. However, if $\mathbf{y}$ lies too low, the right edge $P_{\mathbf{y}}$ might not intersect $\mathcal{C}$. For example, in Figure 5, we have $g_{1}=1$ and $\mathbf{y}$ lies on the line $y_{2}=1$. This this case, $P_{\mathbf{y}}$ contains no integer points and its right edge does not intersect $\mathcal{C}$. Thus, we have no $\mathbf{x}^{\prime}$ and the geometric argument in Figure 4 does not work. However, this can be easily fixed by requiring $a_{0}=g_{1} \geq 2$, noting that AP-COVER is invariant under a simultaneous translation of $J$ and all $\mathrm{AP}_{i}$.

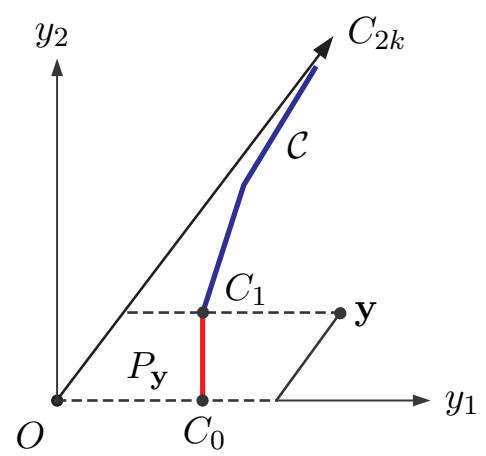

Figure 5 . Here $g_{1}=1, \mathbf{y} \notin \mathcal{C}$, and yet $P_{\mathbf{y}}$ contains no integer points (dotted edges are open).

\footnotetext{
${ }^{2}$ Note that $\mathbf{x}$ and $\mathbf{x}^{\prime}$ are not necessarily two consecutive vertices $C_{i}$ and $C_{i+1}$ of $\mathcal{C}$. They could be two consecutive points on some segment $C_{i} C_{i+1}$.
} 
4.3. Proof of Theorem 1.1 (decision part). Combining (4.3), (4.4) and (4.5), the negation of AP-COVER is equivalent to:

$$
\exists z \in J \quad \forall \mathbf{y} \quad\left[z \not \equiv y_{2}(\bmod M) \vee \mathbf{v} \cdot \mathbf{y}<0 \vee y_{2}<g_{1} \vee \exists \mathbf{x}\left\{\begin{array}{c}
\mathbf{v} \cdot \mathbf{y} \geq \mathbf{v} \cdot \mathbf{x} \geq 0 \\
y_{2}>x_{2}>0
\end{array}\right\}\right]
$$

The condition $z \not \equiv y_{2}(\bmod M)$ can be expressed as:

$$
\exists t \quad 0<z-y_{2}-M t<M .
$$

This existential quantifier $\exists t$ can be absorbed into $\exists \mathbf{x}$ because they are connected by a disjunction. The restricted quantifier $\exists z \in J$ with $J=[\mu, \nu]$ is just

$$
\exists z \quad \mu \leq z \leq \nu .
$$

Overall, we can rewrite (4.6) in prenex normal form:

$$
\begin{aligned}
& \exists z \forall \mathbf{y} \exists \mathbf{x} \mu \leq z \leq \nu \wedge {\left[0<z-y_{2}-M x_{1}<M \vee\right.} \\
&\left.\qquad \mathbf{v} \cdot \mathbf{y}<0 \vee y_{2}<g_{1} \vee\left\{\begin{array}{c}
\mathbf{v} \cdot \mathbf{y} \geq \mathbf{v} \cdot \mathbf{x} \geq 0 \\
y_{2}>x_{2}>0
\end{array}\right\}\right] .
\end{aligned}
$$

All strict inequalities with integer variables can be sharpened. For example $y_{2}>x_{2}$ is equivalent to $y_{2}-1 \geq x_{2}$. This final form contains 5 variables and 10 inequalities.

In summary, we have reduced (the negation of) AP-COVER to (4.7). This shows that (4.7) is NP-hard, and so is $(\text { Short-PA })_{3}$ ). For NP-completeness, by Theorem 3.8 in [Grä87], if (Short-PA ${ }_{3}$ ) is true, there must be a satisfying $\mathbf{z}$ with binary length bounded polynomially in the binary length of $\Phi$. Given such a polynomial length certificate $\mathbf{z}$, one can substitute it into $\left(\right.$ Short-PA ${ }_{3}$ ) and verify the rest of the sentence, which has the form $\forall \mathbf{y} \exists \mathbf{x} \Psi(\mathbf{x}, \mathbf{y})$. Here $\Psi$ is again a short Presburger expression. By Corollary 1.9, this can be checked in polynomial time. Thus, the whole sentence $\left(\right.$ Short-PA $\left._{3}\right)$ is in NP. This concludes the proof of the decision part of Theorem 1.1.

\section{Proof of theorems 1.2 and 1.3 (DECISION PART)}

We will recast (4.7) into the form (GIP). For the polytopes $R$ and $Q$ in (GIP), let $R=J=[\mu, \nu]$ and

$$
Q=\left\{\mathbf{y} \in \mathbb{R}^{2}: y_{2} \geq g_{1}, y_{1} \leq q, \mathbf{v} \cdot \mathbf{y} \geq 0\right\}
$$

see Figure 6 .

Since $R \supset \mathcal{C}^{\prime}$, (4.3) is equivalent to:

$$
\exists z \in R \quad \forall \mathbf{y} \in Q \quad z \not \equiv y_{2}(\bmod M) \vee \mathbf{y} \notin \mathcal{C}^{\prime} .
$$

By condition (4.5), for $\mathbf{y} \in Q$, we have

$$
\mathbf{y} \notin \mathcal{C}^{\prime} \Longleftrightarrow \exists \mathbf{x} \in P_{\mathbf{y}} .
$$

Thus, the sentence (4.7) is equivalent to:

$$
\begin{aligned}
\exists z \in R \quad & \forall \mathbf{y} \in Q \quad \exists \mathbf{x} \\
& 0<z-y_{2}-M x_{1}<M \quad \vee \quad \mathbf{x} \in P_{\mathbf{y}} .
\end{aligned}
$$




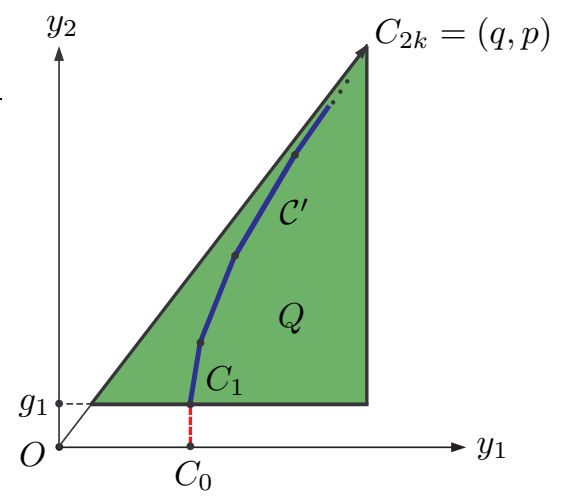

Figure 6. The triangle $Q$ (shaded).

The remaining step is to covert the expression

$$
1 \leq z-y_{2}-M x_{1} \leq M-1 \vee\left\{\begin{array}{l}
\mathbf{v} \cdot \mathbf{y} \geq \mathbf{v} \cdot \mathbf{x} \geq 0 \\
y_{2}-1 \geq x_{2} \geq 1
\end{array}\right\}
$$

into a single system. Here we expanded $\mathbf{x} \in P_{\mathbf{y}}$ and also sharpened all inequalities.

First, observe that for $z \in R$ and $\mathbf{y} \in Q$, there exists $\mathbf{x}$ satisfying (5.3) if and only if there exists such an $\mathbf{x}$ within some bounded range. Indeed, both $R$ and $Q$ are bounded, and (5.3) imply boundedness for $\mathbf{x}$. Therefore, we can take an $N$ large enough so that

$$
-N \leq z, y_{1}, y_{2}, x_{1}, x_{2} \leq N .
$$

For instance, $N=(M+p+q)^{3}$ suffices.

Now we convert (5.3) into a single system. This can be done in two slightly different ways, leading to theorems 1.2 and 1.3 .

5.1. Proof of Theorem $\mathbf{1 . 2}$ (decision part). Applying the distributive law on (5.3), we get an equivalent expression:

$$
\left[\begin{array}{c}
1 \leq z-y_{2}-M x_{1} \leq M-1 \\
\mathbf{v} \cdot \mathbf{x} \leq \mathbf{v} \cdot \mathbf{y}
\end{array}\right] \wedge\left[\begin{array}{c}
1 \leq z-y_{2}-M x_{1} \leq M-1 \\
0 \leq \mathbf{v} \cdot \mathbf{x}
\end{array}\right] \wedge \quad \ldots
$$

Here each $\left[\begin{array}{l}a \\ b\end{array}\right]$ stands for a disjunction $a \vee b$ of two terms. In total, there are four such disjunctions.

Now we convert each of the above disjunctions into a conjunction. WLOG, consider the first one in (5.5). By the bounds (5.4), it is equivalent to:

$$
\left[\begin{array}{c}
1 \leq z-y_{2}-M x_{1} \leq M-1 \\
0 \leq \mathbf{v} \cdot \mathbf{y}-\mathbf{v} \cdot \mathbf{x} \leq 2 N(p+q)
\end{array}\right]
$$

Let $t_{1}=z-y_{2}-M x_{1}$ and $t_{2}=\mathbf{v} \cdot \mathbf{y}-\mathbf{v} \cdot \mathbf{x}$. By (5.4), we always have

$$
\left|t_{1}\right| \leq 2 N+M N, \quad\left|t_{2}\right| \leq 2 N(p+q) .
$$

Define two polygons in $\mathbb{R}^{2}$ :

$$
\begin{gathered}
P_{1}=\left\{\left(t_{1}, t_{2}\right) \in \mathbb{R}^{2}: 1 \leq t_{1} \leq M-1,\left|t_{2}\right| \leq 2 N(p+q)\right\}, \\
P_{2}=\left\{\left(t_{1}, t_{2}\right) \in \mathbb{R}^{2}:\left|t_{1}\right| \leq 2 N+M N, 0 \leq t_{2} \leq 2 N(p+1)\right\} .
\end{gathered}
$$

Then (5.6) can be rewritten as:

$$
\left(t_{1}, t_{2}\right) \in P_{1} \cup P_{2} .
$$


Next, define:

$$
P_{1}^{\prime}=\left(P_{1}, 0\right), \quad P_{2}^{\prime}=\left(P_{2}, 1\right) \quad \text { and } \quad P=\operatorname{conv}\left(P_{1}^{\prime}, P_{2}^{\prime}\right) .
$$

In other words, we embed $P_{1}$ into the plane $t_{3}=0$ and $P_{2}$ into the plane $t_{3}=1$, all inside $\mathbb{R}^{3}$. As 3 -dimensional polytopes, the convex hull of $P_{1}^{\prime}$ and $P_{2}^{\prime}$ is another polytope $P \subset \mathbb{R}^{3}$. It is easy to see that $P$ has 6 facets, whose equations can be found from the vertices of $P_{1}$ and $P_{2}$. Also observe that for $\left(t_{1}, t_{2}, t_{3}\right) \in \mathbb{Z}^{3}$, we have:

$$
\left(t_{1}, t_{2}, t_{3}\right) \in P \Longleftrightarrow \begin{aligned}
& \left(t_{1}, t_{2}\right) \in P_{1}, t_{3}=0, \text { or } \\
& \left(t_{1}, t_{2}\right) \in P_{2}, t_{3}=1 .
\end{aligned}
$$

From this, we have:

$$
\left(t_{1}, t_{2}\right) \in P_{1} \cup P_{2} \Longleftrightarrow \exists t_{3}:\left(t_{1}, t_{2}, t_{3}\right) \in P .
$$

Combined with (5.7), it implies that (5.6) is equivalent to:

$$
\exists t:\left(z-y_{2}-M x_{1}, p y_{1}-q y_{2}-p x_{1}+q x_{2}, t\right) \in P .
$$

The above condition is a linear system with 6 equations. Doing this for each disjunction in (5.5), we get four new variables $\mathbf{t} \in \mathbb{Z}^{4}$ and a combined system of 24 inequalities. Thus, the original disjunction (5.3) is equivalent to a system:

$$
\exists \mathbf{t} \in \mathbb{Z}^{4}: A \mathbf{x}+B \mathbf{y}+C z+D \mathbf{t} \leq \bar{b} .
$$

The inner existential quantifiers $\exists \mathbf{x} \in \mathbb{Z}^{2}$ and $\exists \mathbf{t} \in \mathbb{Z}^{4}$ can be combined into $\exists \mathbf{x} \in \mathbb{Z}^{6}$. Substituting everything into (5.2), we obtain the decision part of Theorem 1.2.

5.2. Proof of Theorem $\mathbf{1 . 3}$ (decision part). Another way to convert (5.3) into a system is to directly interpret its two clauses and two separate polytopes. The same bounds (5.4) still apply. We will need the following special case of the Upper Bound Theorem (see e.g. Theorem 8.23 and Exercise 0.9 in [Zie95]).

Theorem 5.1 (McMullen). A polytope $P \subset \mathbb{R}^{d}$ with $n$ vertices has at most

$$
f(d, n):=\left(\begin{array}{c}
n-\lceil d / 2\rceil \\
n-d
\end{array}\right)+\left(\begin{array}{c}
n-\lfloor d / 2\rfloor-1 \\
n-d
\end{array}\right) \quad \text { facets. }
$$

Similarly, a polytope $Q \subset \mathbb{R}^{d}$ with $n$ facets has at most $f(d, n)$ vertices.

The first polytope we consider is given by:

$$
\left\{\left(x_{1}, y_{2}, z\right) \in \mathbb{R}^{3}: 1 \leq z-y_{2}-M x_{1} \leq M-1,-N \leq x_{1}, y_{2}, z \leq N\right\} .
$$

This is a 3-dimensional polytope with 8 facets. Applying Theorem 5.1, we see that it has at most 12 vertices. To interpret it as a polytope in $z, \mathbf{y}$ and $\mathbf{x}$ we need to form its direct product with the interval $-N \leq y_{2} \leq N$ also embed it in the hyperplane $x_{2}=0$. This produces a polytope $P_{1} \subset \mathbb{R}^{5}$ with 24 vertices.

The second polytope we consider is given by:

$$
\left\{(\mathbf{x}, \mathbf{y}) \in \mathbb{R}^{4}: \mathbf{v} \cdot \mathbf{y} \geq \mathbf{v} \cdot \mathbf{x} \geq 0, y_{2}-1 \geq x_{2} \geq 1, y \in Q\right\} .
$$

As a 4 -dimensional polytope it has only 8 vertices. These 8 vertices correspond to the cases when $\mathbf{y}$ lies at one of the three vertices of $Q$. Two of these vertices give two degenerate parallelograms $P_{\mathbf{y}}$, each of which is a segment with 2 vertices. The lower right vertex of $Q$ gives a non-degenerate parallelogram $P_{\mathbf{y}}$ with 4 vertices. To interpret this as a 5 -dimensional polytope in $z, \mathbf{y}$ and $\mathbf{x}$, we need to form its direct product with the polytope $R=[\mu, \nu]$ for $z$. This results in a polytope $P_{2} \subset \mathbb{R}^{5}$ with 16 vertices. 
Altogether, we have two polytopes $P_{1}, P_{2} \subset \mathbb{R}^{5}$ with 40 vertices in total. We reapply the "lifting" trick in (5.8) to produce another polytope $P \subset \mathbb{R}^{6}$ with 40 vertices so that:

$$
(z, \mathbf{y}, \mathbf{x}) \in P_{1} \cup P_{2} \Longleftrightarrow \exists t:(z, \mathbf{y}, \mathbf{x}, t) \in P .
$$

By Theorem 5.1, the resulting polytope $P$ has at most

$$
f(6,40)=\left(\begin{array}{l}
37 \\
34
\end{array}\right)+\left(\begin{array}{l}
36 \\
34
\end{array}\right)=8400
$$

facets, which can all be found in polynomial time from the vertices. Therefore, the disjunction (5.3) is equivalent to a system:

$$
\exists t: A \mathbf{x}+B \mathbf{y}+C z+D t \leq \bar{b}
$$

with at most 8400 inequalities. The existential quantifiers $\exists t$ and $\exists \mathbf{x} \in \mathbb{Z}^{2}$ can be combined into $\exists \mathbf{x} \in \mathbb{Z}^{3}$. Substituting all into (5.2), we obtain the decision part of Theorem 1.3 .

\section{Proof of theorems 1.1, 1.2 And 1.3 (COUnting Part)}

Notice that the above reduction from AP-COVER to (4.7) is parsimonious, i.e., $z$ lies in $J \backslash\left(\mathrm{AP}_{1} \cup \cdots \cup \mathrm{AP}_{k}\right)$ if and only if $\mu \leq z \leq \nu$ and

$$
\forall \mathbf{y} \exists \mathbf{x}\left[0<z-y_{2}-M x_{1}<M \vee \mathbf{v} \cdot \mathbf{y}<0 \vee y_{2}<g_{1} \vee\left\{\begin{array}{c}
\mathbf{v} \cdot \mathbf{y} \geq \mathbf{v} \cdot \mathbf{x} \geq 0 \\
y_{2}>x_{2}>0
\end{array}\right\}\right]
$$

At the same time, the reduction from 3SAT to AP-COVER given in 9.1 is also parsimonious, i.e., every satisfying assignment $\mathbf{u}$ for (9.1) corresponds to a unique $z \in J$ not covered by the arithmetic progressions and vice versa. This is due the uniqueness part of the Chinese Remainder Theorem used in (9.2). Since \#3SAT is \#P-complete (see e.g. [AB, MM11, Pap94), so is counting the number of $z$ satisfying (6.1). This proves the second part of Theorem 1.1.

The counting parts of theorems 1.2 and 1.3 can be proved with a similar argument to Section 5 .

\section{Proof of Theorem 1.5}

Consider the following $m$-generalization of the problem AP-COVER: 
$m$-AP-COVER

Input: The following elements:

- $m$ intervals $J_{1}=\left[\mu_{1}, \nu_{1}\right], \ldots, J_{m}=\left[\mu_{m}, \nu_{m}\right]$,

- $k_{1}$ triples $\left(g_{1 i}, h_{1 i}, e_{1 i}\right)$, with $1 \leq i \leq k_{1}$, ...

- $k_{m}$ triples $\left(g_{m i}, h_{m i}, e_{m i}\right)$, with $1 \leq i \leq k_{m}$,

- $m$ integers $\tau_{1}, \ldots, \tau_{m} \in \mathbb{Z}$.

Decide:

$$
\begin{array}{rll}
Q_{1}\left(z_{1} \in J_{1} \backslash \Delta_{1}\right) & \ldots & Q_{m-1}\left(z_{m-1} \in J_{m-1} \backslash \Delta_{m-1}\right) \\
& \ldots & Q_{m}\left(z_{m} \in J_{m}\right): \tau_{1} z_{1}+\ldots+\tau_{m} z_{m} \notin \Delta_{m} .
\end{array}
$$

Here $Q_{1}, \ldots, Q_{m} \in\{\forall, \exists\}$ are $m$ alternating quantifiers with $Q_{m}=\exists$.

The sets $\Delta_{1}, \ldots, \Delta_{m}$ are defined as:

$$
\Delta_{t}=\mathrm{AP}_{t 1} \cup \cdots \cup \mathrm{AP}_{t k_{t}}, 1 \leq t \leq m
$$

where

$$
\mathrm{AP}_{t i}=\operatorname{AP}\left(g_{t i}, h_{t i}, e_{t i}\right), 1 \leq i \leq k_{t}
$$

Using Theorem 9.3 , we prove Theorem 1.5 by reducing $m$-AP-COVER to short Presburger arithmetic. Theorem 1.1 is the special case when $m=1\left(\Sigma_{1}^{P} \equiv\right.$ NP). For simplicity, we show the reduction for the case $m=2$. The same argument works for $m>2$.

Consider 2-AP-COVER in (9.6), which is $\Pi_{2}^{P}$-complete. We can rewrite it as:

$$
\forall z_{2} \in J_{2} \quad\left[z_{2} \in \Delta_{2} \quad \vee \exists z_{1} \in J_{1} \tau_{1} z_{1}+\tau_{2} z_{2} \notin \Delta_{1}\right] .
$$

Replacing $z$ with $\tau_{1} z_{1}+\tau_{2} z_{2}$ in (6.1), we can express the condition $\tau_{1} z_{1}+\tau_{2} z_{2} \notin \Delta_{1}$ by a short formula $\forall \mathbf{y} \exists \mathbf{x} \Phi_{1}\left(\mathbf{x}, \mathbf{y}, \tau_{1} z_{1}+\tau_{2} z_{2}\right)$ with 4 extra variables $\mathbf{x}, \mathbf{y} \in \mathbb{Z}^{2}$ and 8 linear inequalities. Similarly, the condition $z_{2} \in \Delta_{2}$ can be expressed as $\exists \mathbf{w} \forall \mathbf{t} \Phi_{2}\left(\mathbf{t}, \mathbf{w}, z_{2}\right)$ with another 4 variables $\mathbf{w}, \mathbf{t} \in \mathbb{Z}^{2}$ and also 8 inequalities.

Overall, (7.1) is equivalent to:

$$
\forall z_{2} \in J_{2} \quad\left[\exists \mathbf{w} \quad \forall \mathbf{t} \Phi_{2}\left(\mathbf{t}, \mathbf{w}, z_{2}\right) \quad \vee \quad \exists z_{1} \in J_{1} \quad \forall \mathbf{y} \quad \exists \mathbf{x} \quad \Phi_{1}\left(\mathbf{x}, \mathbf{y}, \tau_{1} z_{1}+\tau_{2} z_{2}\right)\right] .
$$

Each of the restricted quantifiers $\forall z_{2} \in J_{2}$ and $\exists z_{1} \in J_{1}$ contributes 2 more inequalities. Note that the two quantifier groups $\exists \mathbf{w} \forall \mathbf{t}$ and $\exists z_{1} \forall \mathbf{y} \exists \mathbf{x}$ can be merged through the disjunction into $\exists \mathbf{w} \forall \mathbf{y}^{\prime} \exists \mathbf{x}$. This results in new variables $\mathbf{w} \in \mathbb{Z}^{2}, \mathbf{y}^{\prime}=(\mathbf{t}, \mathbf{y}) \in \mathbb{Z}^{4}$ and $\mathbf{x} \in \mathbb{Z}^{2}$. The final sentence takes the form

$$
\forall z_{2} \quad \exists \mathbf{w} \quad \forall \mathbf{y}^{\prime} \quad \exists \mathbf{x} \quad \Phi\left(\mathbf{x}, \mathbf{y}^{\prime}, \mathbf{w}, z_{2}\right)
$$

with 20 inequalities and 9 variables $\left(z_{1}\right.$ has been absorbed into $\left.\mathbf{w}\right)$.

\section{Bilevel optimization and Pareto optima}

8.1. Proof of Theorem 1.6. First, we characterize the convex chains $\mathcal{C}$ and $\mathcal{D}$ from Figure 1 using a quadratic function:

Lemma 8.1. Let $\alpha=p / q \in \mathbb{Q}_{+}$. If $\mathbf{u}, \mathbf{v} \in \mathbb{Z}^{2}$ satisfy $\frac{u_{2}}{u_{1}}<\alpha<\frac{v_{2}}{v_{1}}$ and $v_{2} u_{1}-v_{1} u_{2}=1$ then both $\frac{u_{2}}{u_{1}}$ and $\frac{v_{2}}{v_{1}}$ are "weak" convergents of $\alpha$, i.e., $\mathbf{u} \in \mathcal{C}$ and $\mathbf{v} \in \mathcal{D}$. 
Proof. Assume $\mathbf{u} \notin \mathcal{C}$, then $\mathbf{u}=\left(u_{1}, u_{2}\right)$ lies stricly below $\mathcal{C}$. By the argument from Lemma 4.1, the parallelogram $P_{\mathbf{u}}$ contains another point $\mathbf{u}^{\prime}=\left(u_{1}^{\prime}, u_{2}^{\prime}\right) \in \mathbb{Z}^{2}$ with $\frac{u_{2}^{\prime}}{u_{1}^{\prime}}<\alpha$. Draw a line $\ell$ parallel to $\overrightarrow{\mathbf{v}}$ and passing through $\mathbf{u}$. Since $\frac{v_{2}}{v_{1}}>\alpha, P_{\mathbf{u}}$ lies completely to the left of $\ell$ (See Figure 7). From this, we conclude that $1=v_{2} u_{1}-v_{1} u_{2}>v_{2} u_{1}^{\prime}-v_{1} u_{2}^{\prime}>0$. In other words, the triangle $O \mathbf{u v}$ has larger area than that of $O \mathbf{u}^{\prime} \mathbf{v}$. This is impossible, because $v_{2} u_{1}^{\prime}-v_{1} u_{2}^{\prime} \in \mathbb{Z}$. Therefore, we must have $\mathbf{u} \in \mathcal{C}$. By the same argument, we have $\mathbf{v} \in \mathcal{D}$.

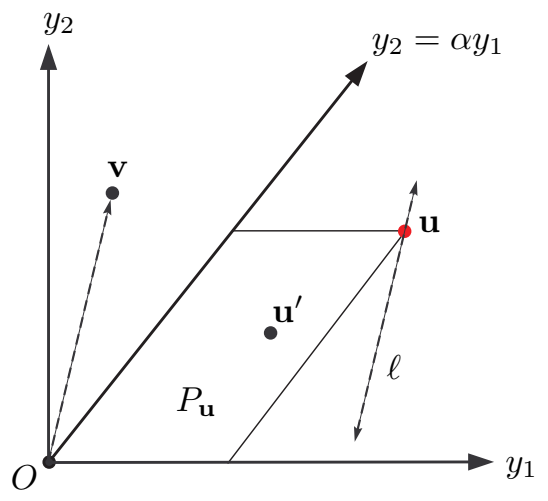

FiguRE 7. u and v.

Conversely, for any weak convergent $\mathbf{u} \in \mathcal{C}$, we can find $\mathbf{v} \in \mathcal{D}$ with $v_{2} u_{1}-v_{1} u_{2}=1$. This comes from the fact that any two consecutive convegents $\frac{p_{i}}{q_{i}}$ and $\frac{p_{i+1}}{q_{i+1}}$ of $\alpha$ satisfy $p_{i+1} q_{i}-p_{i} q_{i+1}=(-1)^{i}$.

Proof of Theorem 1.6. We use the same reduction from AP-COVER as in Sections 4 and 5 . With the same rational number $\alpha=p / q$, let

$$
Q=\left\{\left(u_{1}, u_{2}\right) \in \mathbb{R}^{2}: u_{2} \geq g_{1}, u_{1} \leq q, p u_{1}-q u_{2} \geq 0\right\},
$$

and

$$
P=\left\{\left(v_{1}, v_{2}\right) \in \mathbb{R}^{2}: v_{2} \leq p-1, v_{1} \geq 0, p v_{1}-q v_{2} \leq 0\right\}
$$

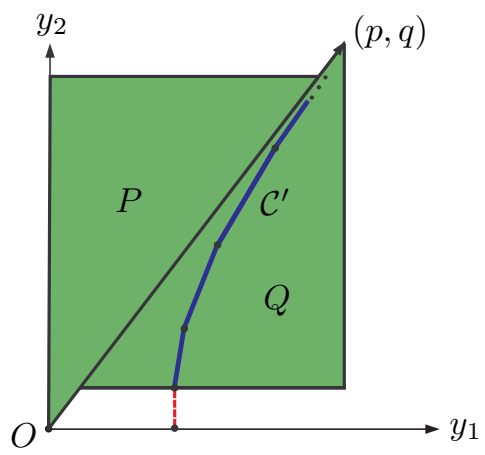

Figure $8 . P$ and $Q$.

Recall from (4.3) that the NP-complete problem AP-COVER asks if there exists some $z \in J \subset[0, M]$ for which no $\mathbf{y} \in \mathcal{C}^{\prime}$ satisfies $z \equiv y_{2}(\bmod M)$. Here $\mathcal{C}^{\prime}$ is the part of the 
convex chain $\mathcal{C}$ lying inside $Q$. Now let $\mathbf{w}=(\mathbf{u}, \mathbf{v}, t), W=Q \times P \times[0, T]$ and

$$
h(z, \mathbf{w})=K\left(v_{2} u_{1}-v_{1} u_{2}-1\right)+\left(u_{2}-z-t M\right)^{2} .
$$

Here $T$ and $K$ are two appropriately chosen constants. Specifically, let $T=p / M$ so that if $z \equiv u_{2}(\bmod M)$ then there always exists $t \in[0, T]$ with $t=\frac{u_{2}-z}{M}$. For $K$, we pick it sufficiently large so that $K \gg\left(u_{2}-z-t M\right)^{2}$ for every $\mathbf{u} \in Q, z \in J$ and $t \in[0, T]$. Clearly $K=(2 T M+p)^{3}$ suffices.

With $\mathbf{u} \in Q \cap \mathbb{Z}^{2}$ and $\mathbf{v} \in P \cap \mathbb{Z}^{2}$, we have $v_{2} u_{1}-v_{1} u_{2} \geq 1$. Furthermore, by Lemma 8.1 , equality happens if and only if $\mathbf{u} \in \mathcal{C}^{\prime}$ and $\mathbf{v} \in \mathcal{D}$. For a fixed $z \in J$ consider the $\mathbf{w} \in W$ that minimizes $h(z, \mathbf{w})$. Since $K \gg\left(z-t M-u_{2}\right)^{2}$, the first term in $h$ always dominate the second one. So we must have $v_{2} u_{1}-v_{1} u_{2}=1$ when $h$ is minimized, which implies $\mathbf{u} \in \mathcal{C}^{\prime}$. Furthermore, among all $\mathbf{y} \in \mathcal{C}^{\prime}, \mathbf{u}$ must be the one for which $u_{2} \bmod M$ is closest to $z$, so that the second term in $h$ is minimized. Thus,

$$
\min _{\mathbf{w} \in W \cap \mathbb{Z}^{5}} h(z, \mathbf{w}) \geq 0,
$$

and equality holds if and only if there is some $\mathbf{y} \in \mathcal{C}^{\prime}$ with $z \equiv y_{2}(\bmod M)$. Therefore,

$$
\max _{z \in J \cap \mathbb{Z}} \min _{\mathbf{w} \in W \cap \mathbb{Z}^{5}} h(z, \mathbf{w})>0
$$

if and only if there exists some $z \in J$ for which no $\mathbf{y} \in \mathcal{C}^{\prime}$ satisfies $z \equiv y_{2}(\bmod M)$. We conclude that computing (1.1) is NP-hard, as it implies AP-COVER.

8.2. Proof of Theorem 1.7. First recall the definition of Pareto optima defined in Section 1.3. To summarize Section 8.1, we showed that computing

$$
\max _{z \in J \cap \mathbb{Z}} \min _{\mathbf{w} \in W \cap \mathbb{Z}^{5}} h(z, \mathbf{w})
$$

is NP-hard for $I \subset \mathbb{R}^{1}$ an interval, $W \subset \mathbb{R}^{5}$ a polytope with 18 facets and $h: \mathbb{R}^{6} \rightarrow \mathbb{R}$ a quadratic function. Let $Q=I \times W \subset \mathbb{R}^{6}$, which has 38 facets. For $\mathbf{x}=(z, \mathbf{w}) \in Q \cap \mathbb{Z}^{6}$, let

$$
f_{1}(\mathbf{x})=z, \quad f_{2}(\mathbf{x})=-z \quad \text { and } \quad f_{3}(\mathbf{x})=h(z, \mathbf{w}) .
$$

Consider the set of Pareto minima of $\left(f_{1}, f_{2}, f_{3}\right)$ on $Q$. For convenience, we denote an outcome vector $\mathbf{y}=\left(f_{1}(\mathbf{x}), f_{2}(\mathbf{x}), f_{3}(\mathbf{x})\right)$ by $\mathbf{y}=f(\mathbf{x})$. Consider two points $\mathbf{x}=(z, \mathbf{w})$ and $\mathbf{x}^{\prime}=\left(z, \mathbf{w}^{\prime}\right)$ in $Q \cap \mathbb{Z}^{6}$. If $h(z, \mathbf{w})<h\left(z, \mathbf{w}^{\prime}\right)$ then $f_{1}(\mathbf{x})=f_{1}\left(\mathbf{x}^{\prime}\right), f_{2}(\mathbf{x})=f_{2}\left(\mathbf{x}^{\prime}\right)$, and $f_{3}(\mathbf{x})<f_{3}\left(\mathbf{x}^{\prime}\right)$. Then $\mathbf{y}^{\prime}=f\left(\mathbf{x}^{\prime}\right)$ is not a Pareto minimum in this case. Therefore, all Pareto minima must be of the form $\mathbf{y}=f(\mathbf{x})$, where $\mathbf{x}=\left(z, \mathbf{w}_{\min }\right)$ with $h\left(z, \mathbf{w}_{\min }\right)=$ $\min _{\mathbf{w} \in W \cap \mathbb{Z}^{5}} h(z, \mathbf{w})$. Furthermore, if $\mathbf{x}=\left(z, \mathbf{w}_{\min }\right)$ and $\mathbf{x}^{\prime}=\left(z^{\prime}, \mathbf{w}_{\min }^{\prime}\right)$ are two such points with $z \neq z^{\prime}$, then the outcome vectors $\mathbf{y}=f(\mathbf{x})$ and $\mathbf{y}^{\prime}=f\left(\mathbf{x}^{\prime}\right)$ are incomparable, simply because either $f_{1}(\mathbf{x})<f_{1}\left(\mathbf{x}^{\prime}\right)$ and $f_{2}(\mathbf{x})>f_{2}\left(\mathbf{x}^{\prime}\right)$, or the other way around.

We conclude that the set Pareto minima of $\left(f_{1}, f_{2}, f_{3}\right)$ on $Q$ is given as:

$$
\mathcal{P}=\left\{\mathbf{y}=\left(z,-z, h\left(z, \mathbf{w}_{\min }\right)\right): z \in J \cap \mathbb{Z}, h\left(z, \mathbf{w}_{\min }\right)=\min _{\mathbf{w} \in W \cap \mathbb{Z}^{5}} h(z, \mathbf{w})\right\} .
$$

For $\mathbf{y} \in \mathbb{R}^{3}$, let $g(\mathbf{y})=-y_{3}$. Then minimizing $g(\mathbf{y})$ over $\mathbf{y} \in \mathcal{P}$ is the same as computing the negated value of (8.1). This proves the first part of Theorem 1.7.

To show the hardness of approximating $\min _{\mathbf{y} \in \mathcal{P}} g(\mathbf{y})$ within a multiplicative factor of $1 / 2$, recall from Section 8.1 that the value of (8.1) determines the AP-COVER. To be precise, (8.1) is equal to the largest squared distance of an integer $z \in J$ from the union $\mathrm{AP}_{1} \cup \cdots \cup \mathrm{AP}_{k}$, which is 0 if and only if $J \cap \mathbb{Z}$ is entirely covered by these APs.

Recall the part of the proof of Theorem 9.1, where we reduce 3SAT to AP-COVER. There, we pick the first $\ell$ primes $p_{1}=2, p_{2}, \ldots, p_{\ell}$. The reduction would work verbatim 
if we picked $p_{2}=3, \ldots, p_{\ell+1}$ instead. The advantage of this small change is that now we can exclude the arithmetic progression $z \equiv 0(\bmod 2)$ from $J$. In other words, we require $z \equiv 1(\bmod 2)$ and the Chinese Remainder Theorem still works. Then the final union $\mathrm{AP}_{1} \cup \cdots \cup \mathrm{AP}_{k}$ which we exclude from $J$ must contain all even numbers. This implies that the largest squared distance of an integer $z \in J$ to $\mathrm{AP}_{1} \cup \cdots \cup \mathrm{AP}_{k}$ is at most 1 . Therefore, the value of (8.1) is either 1 or 0 . So getting a $1 / 2$-approximation is equivalent to deciding AP-COVER, and thus NP-hard.

\section{COVERING With ARITHMETIC PROGRESSIONS}

9.1. NP-completeness of AP-COVER. Recall the following problem from 4.1 ,

\section{AP-COVER}

Input: $\quad$ An interval $J=[\mu, \nu] \subset \mathbb{Z}$ and $k$ triples $\left(g_{i}, h_{i}, e_{i}\right)$ for $i=1, \ldots, k$.

Decide: Is there $z \in I$ such that $z \notin\left(\mathrm{AP}_{1} \cup \cdots \cup \mathrm{AP}_{k}\right)$, where $\operatorname{AP}_{i}=\operatorname{AP}\left(g_{i}, h_{i}, e_{i}\right)$ ?

In this section, we reproduce (in a somewhat different language) the original proof from [SM73, see also Remark 9.2 below. The reduction in the proof will later be extended to work with more quantifiers.

Theorem 9.1 (Stockmeyer and Meyer). AP-COVER is NP-complete.

Proof. We reduce 3SAT to AP-COVER. Consider a 3-CNF Boolean expression:

$$
\Psi(\mathbf{u})=\bigwedge_{i=1}^{n} C_{i}(\mathbf{u}),
$$

where $\mathbf{u}=u_{1} \ldots u_{\ell} \in\{\text { true, false }\}^{\ell}$ are Boolean variables, and each clause $C_{i}(\mathbf{u})$ is a disjunction of three literals from the set

$$
\left\{u_{j}, \neg u_{j}: 1 \leq j \leq \ell\right\} .
$$

Let $p_{1}, \ldots, p_{\ell}$ be the first $\ell$ primes. We have $p_{\ell}=O(\ell \log \ell)$ by the Prime Number Theorem. So $p_{1}, \ldots, p_{\ell}$ can be found in time poly $(\ell)$. We restrict $z$ to the interval $J=[0, p)$, where $p=p_{1} \cdots p_{\ell}$. For each assignment of $\mathbf{u}=u_{1} \ldots u_{\ell} \in\{\text { true, false }\}^{\ell}$, we shall associate a unique integer $z \in J$ that satisfies:

$$
u_{j}=\text { true } \Longleftrightarrow z \equiv 1\left(\bmod p_{j}\right) \quad ; \quad u_{j}=\text { false } \Longleftrightarrow z \equiv 0\left(\bmod p_{j}\right) .
$$

First, for each $j$, we exclude all $\operatorname{moduli} \bmod p_{j}$ that are not 0 or 1 . In other words, we exclude the arithmetic progressions:

$$
\mathrm{AP}_{j t}=\left\{z \in J: z \equiv t\left(\bmod p_{j}\right)\right\} \quad \text { for } \quad 1 \leq j \leq \ell, 2 \leq t<p_{j} .
$$

If $z \notin \bigcup_{j t} \mathrm{AP}_{j t}$ then $z$ is equal to 0 or $1 \bmod$ every $p_{j}$. Now consider each clause $C_{i}(\mathbf{u})$. For example, assume $C_{1}(\mathbf{u})=u_{1} \vee \neg u_{2} \vee u_{3}$. The negation $\neg C_{1}(\mathbf{u})$ is $\neg u_{1} \wedge u_{2} \wedge \neg u_{3}$. To this, we associate an arithmetic progression:

$$
\mathrm{AP}_{1}=\left\{z \in J: z \equiv 0\left(\bmod p_{1}\right) \wedge z \equiv 1\left(\bmod p_{2}\right) \wedge z \equiv 0\left(\bmod p_{3}\right)\right\} .
$$

By the Chinese remainder theorem, we can write:

$$
\mathrm{AP}_{1}=\left\{z \in J: z \equiv e\left(\bmod p_{1} p_{2} p_{3}\right)\right\},
$$

where $e$ is unique mod $p_{1} p_{2} p_{3}$ and also computable in polynomial time. Then we have:

$$
C_{1}(\mathbf{u})=\text { true } \quad \Longleftrightarrow \quad z \notin \mathrm{AP}_{1} \text {. }
$$


Doing this for all clauses $C_{1}, \ldots, C_{n}$, we get $n$ arithmetic progressions $\mathrm{AP}_{1}, \ldots, \mathrm{AP}_{n}$. From (9.1), (9.3) and (9.5), we conclude that:

$$
\Psi(\mathbf{u})=\bigwedge_{i=1}^{n} C_{i}(\mathbf{u})=\text { true } \Longleftrightarrow z \notin \bigcup_{1 \leq i \leq n} \operatorname{AP}_{i} \bigcup_{\substack{1 \leq j \leq \ell \\ 2 \leq t<p_{j}}} \mathrm{AP}_{j t} .
$$

Therefore,

$$
\exists \mathbf{u} \quad \Psi(\mathbf{u})=\text { true } \Longleftrightarrow \exists z \in J \quad: \quad z \notin \bigcup_{1 \leq i \leq n} \mathrm{AP}_{i} \bigcup_{\substack{1 \leq j \leq \ell \\ 2 \leq t<p_{j}}} \operatorname{AP}_{j t} .
$$

The above LHS is a 3SAT sentence, which is NP-complete to decide. Thus, the RHS, which is AP-COVER, is also NP-complete. In total, we have $k:=n+\sum_{j=1}^{\ell}\left(p_{j}-1\right)$ arithmetic progressions, each of which can be given as a triple $\left(g_{i}, h_{i}, e_{i}\right)$.

Remark 9.2. In [GJ79, §A7], the problem AP-COVER is phrased differently under the name SIMULTANEOUS INCONGRUENCES problem.

9.2. Generalization of AP-COVER to $m$ quantifiers. We consider the following $m$ generalization of the problem AP-COVER.

$m$-AP-COVER

Input: The following elements:

- $m$ intervals $J_{1}=\left[\mu_{1}, \nu_{1}\right], \ldots, J_{m}=\left[\mu_{m}, \nu_{m}\right]$,

- $k_{1}$ triples $\left(g_{1 i}, h_{1 i}, e_{1 i}\right)$, with $1 \leq i \leq k_{1}$,

$\ldots$

- $k_{m}$ triples $\left(g_{m i}, h_{m i}, e_{m i}\right)$, with $1 \leq i \leq k_{m}$,

- $m$ integers $\tau_{1}, \ldots, \tau_{m} \in \mathbb{Z}$.

Decide: The truth of the sentence:

$$
\begin{array}{rll}
Q_{1}\left(z_{1} \in J_{1} \backslash \Delta_{1}\right) & \ldots Q_{m-1}\left(z_{m-1} \in J_{m-1} \backslash \Delta_{m-1}\right) \\
& \ldots Q_{m}\left(z_{m} \in J_{m}\right): \tau_{1} z_{1}+\ldots+\tau_{m} z_{m} \notin \Delta_{m} .
\end{array}
$$

Here $Q_{1}, \ldots, Q_{m} \in\{\forall, \exists\}$ are $m$ alternating quantifiers with $Q_{m}=\exists$. The sets $\Delta_{1}, \ldots, \Delta_{m}$ are defined as:

$$
\Delta_{t}=\mathrm{AP}_{t 1} \cup \cdots \cup \mathrm{AP}_{t k_{t}}, 1 \leq t \leq m
$$

where

$$
\mathrm{AP}_{t i}=\mathrm{AP}\left(g_{t i}, h_{t i}, e_{t i}\right), 1 \leq i \leq k_{t}
$$

For example, 2-AP-COVER asks whether

$$
\forall\left(z_{2} \in J_{2} \backslash \Delta_{2}\right) \quad \exists z_{1} \in J_{1} \quad \tau_{1} z_{1}+\tau_{2} z_{2} \notin \Delta_{1},
$$

i.e., for all $z_{2} \in J_{2}$ either $z_{2}$ is covered by some AP in the first group, or there is some $z_{1} \in J_{1}$ so that their linear combination $\tau_{1} z_{1}+\tau_{2} z_{2}$ is not covered by any AP in the second group.

Theorem 9.3. $m$-AP-COVER is $\Sigma_{m}^{\mathrm{P}}$-complete for $m$ odd and $\Pi_{m}^{\mathrm{P}}$-complete for $m$ even.

Proof. For simplicity, we show that 2-AP-COVER is $\Pi_{2}^{\mathrm{P}}$-complete. The proof for general $m$-AP-COVER is analogous. 
This is similar to Theorem 9.1 s proof, but instead of 3SAT we decide:

$$
\forall \mathbf{v} \quad \exists \mathbf{u} \quad \Psi(\mathbf{u}, \mathbf{v})=\text { true, }
$$

where $\mathbf{u}, \mathbf{v} \in\{\text { true, false }\}^{\ell}$, and $\Psi(\mathbf{u}, \mathbf{v})=\bigwedge_{i=1}^{n} C_{i}(\mathbf{u}, \mathbf{v})$, with each clause $C_{i}(\mathbf{u}, \mathbf{v})$ a disjunction of three literals from the set

$$
\left\{u_{j}, \neg u_{j}, v_{j}, \neg v_{j}: 1 \leq j \leq \ell\right\} .
$$

Deciding (9.7) is $\Pi_{2}^{\mathrm{P}}$-complete (see e.g. GJ79, Pap94). To reduce (9.7) to (9.6), we again take the first $2 \ell$ primes $p_{1}, \ldots, p_{\ell}, q_{1}, \ldots, q_{\ell}$. Let $p=p_{1} \cdots p_{\ell}, q=q_{1} \cdots q_{\ell}$ and:

$$
J_{1}:=[0, p) \text { and } J_{2}:=[0, q) .
$$

Since $\operatorname{gcd}(p, q)=1$, we can also find in polynomial time $\tau_{1}, \tau_{2} \in \mathbb{Z}$ so that:

$$
\tau_{1} \equiv 1(\bmod p), q \mid \tau_{1} \quad \text { and } \tau_{2} \equiv 1(\bmod q), p \mid \tau_{2} .
$$

Next, we require that $z_{2} \equiv 0$ or $1\left(\bmod q_{j}\right)$ for $i=1, \ldots, \ell$. This can be expressed as $z_{2} \in J_{2} \backslash \Delta_{2}$, where $\Delta_{2}$ is a union of some arithmetic progressions similar to those in (9.3). These are the $k_{2}$ progressions $\mathrm{AP}_{21}, \ldots, \mathrm{AP}_{2 k_{2}}$.

We also require $z_{1} \equiv 0$ or $1\left(\bmod p_{j}\right)$ for $j=1, \ldots, \ell$. By $(9.8)$, this is equivalent to $\tau_{1} z_{1}+\tau_{2} z_{2} \equiv 0$ or $1\left(\bmod p_{j}\right)$. Again, this condition can be expressed as:

$$
\tau_{1} z_{1}+\tau_{2} z_{2} \notin \Gamma_{1}
$$

for $\Gamma_{1}$ a union of some arithmetic progressions.

Analogous to (9.2), the variables $z_{1}$ and $z_{2}$ correspond to $\mathbf{u}$ and $\mathbf{v}$, respectively. By the Chinese remainder theorem (see (9.4) and (9.5)), we can express each clause $C_{i}(\mathbf{u}, \mathbf{v})$ as:

$$
C_{1}(\mathbf{u}, \mathbf{v})=\text { true } \Longleftrightarrow \tau_{1} z_{1}+\tau_{2} z_{2} \notin \mathrm{AP}_{i}
$$

for some arithmetic progression $\mathrm{AP}_{i}$ with $i=1, \ldots, n$. Let $\Delta_{1}$ be the union of $\Gamma_{1}$ in (9.9) with $\mathrm{AP}_{1}, \ldots, \mathrm{AP}_{n}$.

Overall, we have $k_{1}+k_{2}$ finite arithmetic progressions from $\Delta_{1}$ and $\Delta_{2}$. Note that $k_{1}+k_{2}$ is still polynomial compared to $\ell$ and the length of $\Psi$. It is straightforward that (9.6) and (9.7) are equivalent. Therefore, deciding (9.6) is $\Pi_{2}^{\mathrm{P}}$-complete.

\section{On Kannan's Partition Theorem}

10.1. Validity of KPT. By Parametric Integer Programming (PIP), we mean the following problem. Given an integer matrix $A \in \mathbb{Z}^{m \times n}$ and a $k$-dimensional polyhedron $W \subset \mathbb{R}^{m}$, is the following sentence true:

$$
\forall \bar{b} \in W \quad \exists \mathbf{x} \in \mathbb{Z}^{n} \quad: \quad A \mathbf{x} \leq \bar{b} .
$$

We think of $\bar{b}$ as a parameter varying over $W$. For every fixed $\bar{b}$, this gives an Integer Programming problem in fixed dimension $n$. In [Kan90, Theorem 3.1], Kannan claimed the following result, which implies a polynomial time algorithm to decide (10.1). From here on, we use RA to denote rational affine transformations. Also let $K_{\bar{b}}:=\left\{\mathbf{x} \in \mathbb{R}^{n}: A \mathbf{x} \leq \bar{b}\right\}$ for every $\bar{b} \in W$.

Theorem 10.1 (Kannan's Partition Theorem). Fix $n$ and $k$. Given a PIP problem, we can find in polynomial time a partition

$$
W=P_{1} \sqcup P_{2} \sqcup \cdots \sqcup P_{r},
$$


where each $P_{i}$ is a rational copolyhedron ${ }^{3}$, so that the partition satisfies the following properties. For each $P_{i}$, we can find in polynomial time a finite set $\mathcal{T}_{i}=\left\{\left(S_{i j}, T_{i j}\right)\right\}$ of pairs of $R A s T_{i j}: \mathbb{R}^{m} \rightarrow \mathbb{R}^{n}$ and $S_{i j}: \mathbb{Z}^{n} \rightarrow \mathbb{Z}^{n}$, so that for every $\bar{b} \in P_{i}$ we have:

$$
K_{\bar{b}} \cap \mathbb{Z}^{n} \neq \varnothing \Longleftrightarrow \exists\left(S_{i j}, T_{i j}\right) \in \mathcal{T}_{i}: S_{i j}\left\lfloor T_{i j} \bar{b}\right\rfloor \in K_{\bar{b}}
$$

Furthermore, for each $P_{i}$, the set $\mathcal{T}_{i}$ contains at most $n^{4 n}$ pairs $\left(S_{i j}, T_{i j}\right)$. The number of all $P_{i}$ is $r \leq(m n \phi)^{k n^{\delta n}}$, where $\phi$ is the binary length of $A$ and $\delta$ is a universal constant.

KPT claims that in order to solve for an $\mathbf{x} \in \mathbb{Z}^{n}$ satisfying $A \mathbf{x} \leq \bar{b}$ with $\bar{b}$ varying over $W$, we only need to preprocess the matrix $A$ in polynomial time and obtain a polynomial number of regions $P_{i}$. When queried with $\bar{b} \in P_{i}$, we only need to check for a fixed number $\left(n^{4 n}\right)$ of candidates of the form $\mathbf{x}=S_{i j}\left\lfloor T_{i j} \bar{b}\right\rfloor$ to get an integer solution in $K_{\bar{b}}$ (if any exists).

Let us prove that KPT, if true, would imply far stronger statements for a PIP problems that involves only a matrix of fixed length $m$. From now on, fix $m, n$ and $k$. By KPT and the observation $m n \leq \phi$, the number of regions $P_{i}$ in (10.2) can be bounded as:

$$
r \leq(m n \phi)^{k n^{\delta n}} \leq \phi^{\gamma(n, k)} .
$$

Here $\gamma(n, k)$ is a constant which depends only on $n$ and $k$. The following structural result is an implication of KPT when the parameter space $W$ is 1-dimensional, i.e. when $k=1$ :

$$
W=\left\{f(y) \in \mathbb{R}^{m}: y \in I\right\}
$$

where $f: \mathbb{R}^{1} \rightarrow \mathbb{R}^{m}$ is a $\mathrm{RA}$, and $I \subset \mathbb{R}$ a bounded interval.

Lemma 10.2. Assume (10.3) holds. Given a PIP problem with a 1-dimensional parameter space $W$ (10.4), there exists a finite set $\mathcal{T}=\left\{\left(S_{j}, T_{j}\right)\right\}$ of pairs of $R A s T_{j}: \mathbb{R}^{1} \rightarrow \mathbb{R}^{n}$ and $S_{j}: \mathbb{Z}^{n} \rightarrow \mathbb{Z}^{n}$ so that the following hold. For every $y \in I \cap \mathbb{Z}$ and $\bar{b}=f(y) \in \mathbb{R}^{m}$, we have:

$$
K_{\bar{b}} \cap \mathbb{Z}^{n} \neq \varnothing \quad \Longleftrightarrow \quad \exists\left(S_{j}, T_{j}\right) \in \mathcal{T}: S_{j}\left\lfloor T_{j} y\right\rfloor \in K_{\bar{b}} .
$$

Furthermore, the set $\mathcal{T}$ contains at most $c(n)$ pairs $\left(S_{j}, T_{j}\right)$, where $c(n)$ is a constant which depends only on $n$.

Remark 10.3. The above lemma says that the bound (10.3) as implied by KPT would guarantee a small set of candidates for any "short" PIP problem $A \mathbf{x} \leq f(y)$ with 1-dimensional parameters $y$. The number of candidates $c(n)$ depends only on the dimension $n$.

Proof of Lemma 10.2. WLOG, assume $I=[0, N)$ and $A=\left(a_{i j}\right) \in \mathbb{Z}^{m \times n}$. Let

$$
M=N \prod_{i j}\left(\left|a_{i j}\right|+1\right) \prod_{k}\left(\left|p_{k} q_{k}\right|+1\right)
$$

where $p_{k} / q_{k}$ runs over all rational coefficients in $f$. Let $J=[0, M N)$. Consider the following PIP problem with one parameter $y^{\prime} \in J$ and $n+2$ integer variables $\mathbf{x} \in \mathbb{Z}^{n}, y_{1}, y_{2} \in \mathbb{Z}$ :

$$
N y_{1}+y_{2}=y^{\prime}, \quad 0 \leq y_{1}<M, \quad 0 \leq y_{2}<N, \quad A \mathbf{x}-f\left(y_{2}\right) \leq 0 .
$$

Observe that when (10.6) is feasible, the values of $y_{1}$ and $y_{2}$ are uniquely determined. Indeed, we should have $y_{1}=\left\lfloor y^{\prime} / N\right\rfloor$ and $y_{2}=y^{\prime}-N y_{1}$. So as $y^{\prime}$ varies over $J \cap \mathbb{Z}$, the solutions of (10.6) correspond bijectively with the solutions of the original PIP problem $A \mathbf{x} \leq f(y)$ where $y=\left\lfloor y^{\prime} / N\right\rfloor \in I$.

Clearly, (10.6) can be put into the form $B \mathbf{z} \leq g\left(y^{\prime}\right)$ where $\mathbf{z}=\left(\mathbf{x}, y_{1}, y_{2}\right) \in \mathbb{Z}^{n+2}$ are variables and $g$ is an RA. Let $\bar{b}^{\prime}=g\left(y^{\prime}\right)$, then the problem takes the form $B \mathbf{z} \leq \bar{b}^{\prime}$. Also let

\footnotetext{
${ }^{3} \mathrm{~A}$ copolyhedron is a convex polyhedron with possibly some open facets.
} 
$W^{\prime}=\left\{\bar{b}^{\prime}=g\left(y^{\prime}\right): y^{\prime} \in J\right\}$. Applying KPT to the PIP problem $B \mathbf{z} \leq \bar{b}^{\prime}$ with a 1-dimensional parameter space $W^{\prime}$, we have a partition of $W^{\prime}$ into polynomially many intervals. Since $\bar{b}^{\prime}=g\left(y^{\prime}\right)$ and $g$ is an RA, this partition induces another partition on $J$ (the space for $y^{\prime}$ ) into intervals:

$$
J=J_{1} \sqcup \cdots \sqcup J_{r}
$$

By (10.3), the number $r$ of all intervals in this partition is polynomial in the binary length of the matrix $B$. From (10.5) and (10.6), it is clear that $B$ has no more than $2 m n$ entries, each bounded by $M$. Therefore, we have:

$$
r \leq\left(\sum_{i j}\left\lceil\log b_{i j}\right\rceil\right)^{\gamma} \leq(2 m n \log M)^{\gamma} \ll M .
$$

Here $\gamma=\gamma(n, k)$ is some constant degree guaranteed by KPT. Since $r \ll M$, some interval $J_{i}$ from (10.7) must contain an entire subinterval $I^{\prime}=[k N,(k+1) N)$ for some $0 \leq k<M$. For simplicity, assume $I^{\prime}=[k N,(k+1) N] \subseteq J_{1}$.

Also by KPT, for the interval $J_{1}$, there is a set of candidates $\mathcal{T}_{1}=\left\{\left(S_{1 j}, T_{1 j}\right)\right\}$ of size at most $c(n):=(n+2)^{4(n+2)}$ for the PIP problem $B \mathbf{z} \leq \bar{b}^{\prime}$. For every $y^{\prime} \in I^{\prime} \subseteq J_{1}$, each solution of (10.6) should have $y_{1}=k$ and $y_{2}=y^{\prime}-N k$. By a translation $y=y^{\prime}-N k$, we can map $I^{\prime}$ back to $I$. Accordingly, we can modify each candidate $\left(S_{i j}, T_{i j}\right) \in \mathcal{T}_{i}$ to be a pair of RAs in $y$. Clearly, they serve as candidates for the original PIP problem $A \mathbf{x} \leq f(y)$ with $y \in I$.

Lemma 10.2 can be easily boosted to a $k$-dimensional parameter space $W$ for a fixed $k$ :

$$
W=\left\{f(\mathbf{y}) \in \mathbb{R}^{m}: \mathbf{y} \in R\right\}
$$

with $f: \mathbb{R}^{k} \rightarrow \mathbb{R}^{m}$ an $\mathrm{RA}$ and $R \subset \mathbb{R}^{k}$ a rectangular box.

Lemma 10.4. Assume (10.3) holds. Given a PIP problem with a $k$-dimensional parameter space $W$ (10.9), there exists a finite set $\mathcal{T}=\left\{\left(S_{j}, T_{j}\right)\right\}$ of pairs of $R A s T_{j}: \mathbb{R}^{k} \rightarrow \mathbb{R}^{n}$ and $S_{j}: \mathbb{Z}^{n} \rightarrow \mathbb{Z}^{n}$ so that the following hold. For every $\mathbf{y} \in R \cap \mathbb{Z}^{k}$ and $\bar{b}=f(\mathbf{y}) \in \mathbb{R}^{m}$, we have:

$$
K_{\bar{b}} \cap \mathbb{Z}^{n} \neq \varnothing \quad \Longleftrightarrow \quad \exists\left(S_{j}, T_{j}\right) \in \mathcal{T}: S_{j}\left\lfloor T_{j} \mathbf{y}\right\rfloor \in K_{\bar{b}} .
$$

Furthermore, the set $\mathcal{T}$ contains at most $c(n, k)$ pairs $\left(S_{j}, T_{j}\right)$, where $c(n, k)$ is a constant which depends only on $n$ and $k$.

Proof. WLOG, assume $R=\left[0, r_{1}\right) \times \ldots \times\left[0, r_{k}\right)$. We "flatten" the $k$-dimensional parameter $\mathbf{y}$. For every $\mathbf{y}=\left(y_{1}, \ldots, y_{k}\right) \in R$, let:

$$
y^{\prime}=y_{1}+y_{2} r_{1}+y_{3}\left(r_{1} r_{2}\right)+\ldots+y_{k}\left(r_{1} \cdots r_{k-1}\right) \in\left[0, r_{1} \cdots r_{k}\right) .
$$

This RA maps the integer points in $R$ bijectively to those in $I=\left[0, r_{1} \cdots r_{k}\right)$. We rewrite $A \mathbf{x} \leq f(\mathbf{y})$ as another PIP problem with a 1-dimensional parameter $y^{\prime} \in I$ and $n+k$ variables $\mathbf{x} \in \mathbb{Z}^{n}, \mathbf{y} \in \mathbb{Z}^{k}$ :

$$
\begin{gathered}
y^{\prime}=y_{1}+y_{2} r_{1}+y_{3}\left(r_{1} r_{2}\right)+\ldots+y_{k}\left(r_{1} \cdots r_{k-1}\right), \\
0 \leq y_{i}<r_{i} \text { for } 1 \leq i \leq k, \quad A \mathbf{x}-f(\mathbf{y}) \leq 0 .
\end{gathered}
$$

Note that (10.11) has a solution if and only if the original PIP problem $A \mathbf{x} \leq f(\mathbf{y})$ has a solution. Furthermore, in every solution of (10.11), the variables $\mathbf{y}$ are uniquely determined by $y^{\prime}$ via the RA (10.10). Applying Lemma 10.2, we get a set $\mathcal{T}^{\prime}=\left\{\left(S_{j}^{\prime}, T_{j}^{\prime}\right)\right\}$ of at most $c(n, k):=(n+k+2)^{4(n+k+2)}$ candidates for (10.11), where $T_{j}^{\prime}: \mathbb{R}^{1} \rightarrow \mathbb{R}^{n+k}$ and 
$S_{j}^{\prime}: \mathbb{Z}^{n+k} \rightarrow \mathbb{Z}^{n+k}$ are pairs of RAs. Using (10.10), we can re-express each pair $\left(S_{j}^{\prime}, T_{j}^{\prime}\right)$ as a pair $\left(S_{j}, T_{j}\right)$ with $T_{j}: \mathbb{R}^{k} \rightarrow \mathbb{R}^{n}$ and $S_{j}: \mathbb{Z}^{n} \rightarrow \mathbb{Z}^{n}$ so that (10.11) has a solution if and only if $\mathbf{x}=S_{j}\left\lfloor T_{j} \mathbf{y}\right\rfloor$ satisfies $A \mathbf{x} \leq f(\mathbf{y})$ for some $j$. In other words, $\mathcal{T}=\left\{\left(S_{j}, T_{j}\right)\right\}$ is a finite set of at most $c(n, k)$ candidates for the original PIP problem $A \mathbf{x} \leq f(\mathbf{y})$.

Remark 10.5. Since the dimensions of $A$ are fixed, each condition $S_{i j}\left\lfloor T_{i j} \mathbf{y}\right\rfloor \in K_{\bar{b}}$ can be expressed as a short Boolean combination of linear inequalities, at the cost of introducing a few extra $\exists$ or $\forall$ quantifiers. For example, a condition $\frac{1}{2}+\lfloor y / 5\rfloor \leq 3$ for $y \in \mathbb{Z}$ can be expressed as either

$$
\exists t\left\{\begin{array}{ccc}
t & \leq & y / 5 \\
t & > & y / 5-1 \\
\frac{1}{2}+t & \leq & 3
\end{array}\right\} \quad \text { or } \quad \forall t\left[\begin{array}{ccc}
t & > & y / 5 \\
t & \leq & y / 5-1 \\
\frac{1}{2}+t & \leq & 3
\end{array}\right] .
$$

Here $\{\cdot\}$ is a conjunction and [.] is a disjunction.

Now we relax the parameter space $W$ to an arbitrary $k$-dimensional polyhedron, i.e.,

$$
W=\left\{f(\mathbf{y}) \in \mathbb{R}^{m}: \mathbf{y} \in Q\right\}
$$

with $f: \mathbb{R}^{k} \rightarrow \mathbb{R}^{m}$ an $\mathrm{RA}$ and $Q \subset \mathbb{R}^{k}$ a polyhedron.

Corollary 10.6. Assume (10.3) holds. Then for every fixed $m, n$ and $k$, there is a constant $d(m, n, k)$ so that the following holds. For a PIP problem with a $k$-dimensional parameter space $W$ (10.13), let:

$$
Q^{\prime}=\left\{\mathbf{y} \in Q \cap \mathbb{Z}^{k}: A \mathbf{x} \leq f(\mathbf{y}) \text { has no solutions } \mathbf{x} \in \mathbb{Z}^{n}\right\} .
$$

If $\left|Q^{\prime}\right|>d(m, n, k)$, then it contains three distinct points $\mathbf{y}_{1}, \mathbf{y}_{2}, \mathbf{y}_{3}$ with $\mathbf{y}_{3}=\left(\mathbf{y}_{1}+\mathbf{y}_{2}\right) / 2$.

Proof. Let $R$ be a large enough box that contains $Q$. Applying Lemma 10.4 to the PIP problem $A \mathbf{x} \leq f(\mathbf{y})$ with $\mathbf{y} \in R$, we get a set of candidates $\mathcal{T}=\left\{\left(S_{j}, T_{j}\right)\right\}$ of size at most $c(n, k)$ so that:

$$
A \mathbf{x} \leq f(\mathbf{y}) \text { has no solutions } \quad \Longleftrightarrow \quad \forall\left(S_{j}, T_{j}\right) \in \mathcal{T}: S_{j}\left\lfloor T_{j} \mathbf{y}\right\rfloor \not \leq f(\mathbf{y}) .
$$

By the argument in Remark 10.5, each condition $S_{j}\left\lfloor T_{j} \mathbf{y}\right\rfloor \not \leq f(\mathbf{y})$ can be expressed by a short Presburger formula $\exists \mathbf{t} \Phi_{j}(\mathbf{y}, \mathbf{t})$ with length bounded in $m$ (fixed). Taking conjunction over all such formulas for $1 \leq j \leq c(n, k)$, we have:

$$
A \mathbf{x} \leq f(\mathbf{y}) \text { has no solutions } \Longleftrightarrow \quad \exists \widetilde{\mathbf{t}} \quad \Phi(\mathbf{y}, \widetilde{\mathbf{t}}) \text { 者 }
$$

Here $\Phi$ is still a short Presburger expression in a bounded number of variables. Denote by $\lambda$ and $\mu$ the total number of variables and inequalities in $\Phi$, respectively. Both of these are constants in $m, n$ and $k$. Let $d=d(m, n, k)=2^{\lambda+\mu}$. The $\mu$ inequalities in $\Phi$ determine $\mu$ hyperplanes in $\mathbb{R}^{\lambda}$. These hyperplanes partition $\mathbb{R}^{\lambda}$ into polyhedral regions:

$$
\mathbb{R}^{\lambda}=W_{1} \sqcup \cdots \sqcup W_{\eta},
$$

with $\eta \leq 2^{\mu}$. Observe that as $(\mathbf{y}, \widetilde{\mathbf{t}})$ varies over a single region $W_{j}$, the value of $\Phi(\mathbf{y}, \widetilde{\mathbf{t}})$ is always true or always false. Since $\left|Q^{\prime}\right|>d$, we have at least $d+1$ distinct pairs $\left(\mathbf{y}_{1}, \widetilde{\mathbf{t}}_{1}\right), \ldots,\left(\mathbf{y}_{d+1}, \widetilde{\mathbf{t}}_{d+1}\right)$ for each of which $\Phi\left(\mathbf{y}_{i}, \widetilde{\mathbf{t}}_{i}\right)=$ true. By the pigeon hole principle, some region $W_{j}$ contains at least $2^{\lambda}+1$ of these pairs. Each such pair is a point in $\mathbb{Z}^{\lambda}$, so at least two of them must have coordinates equal mod 2 pairwise. Assume $\left(\mathbf{y}_{1}, \widetilde{\mathbf{t}}_{1}\right)$ and $\left(\mathbf{y}_{2}, \widetilde{\mathbf{t}}_{2}\right)$ are two such two pairs. By convexity, $\left(\mathbf{y}_{1}+\mathbf{y}_{2}, \widetilde{\mathbf{t}}_{1}+\widetilde{\mathbf{t}}_{2}\right) / 2$ is another integer

\footnotetext{
${ }^{4}$ Separate variables $\mathbf{t}$ for different $\Phi_{j}$ must be concatenated into $\widetilde{\mathbf{t}}$.
} 
point in $W_{j}$. Since $\Phi$ is always true over $W_{j}$, this pair also satisfies $\Phi$. By (10.14), the point $\mathbf{y}_{3}=\left(\mathbf{y}_{1}+\mathbf{y}_{2}\right) / 2$ also lies in $Q^{\prime}$. We conclude that $\mathbf{y}_{1}, \mathbf{y}_{2}, \mathbf{y}_{3} \in Q^{\prime}$.

Theorem 10.7. The bound (10.3) as claimed by KPT does not hold in full generality. In other words, even for $k=1$ and fixed $m, n$, the number of pieces $r$ in the partition (10.2) must be at least $\exp (\varepsilon \phi)$ for some constant $\varepsilon=\varepsilon(m, n)>0$.

Proof. Assume (10.3) holds. Consider the following continued fraction of length $(2 s+1)$ :

$$
\alpha_{s}=[2 ; 1, \ldots, 1]=p / q,
$$

where $p=F_{2 s+3}, q=F_{2 s+1}$ are the Fibonacci numbers. From Properties (G1)-(G6) in Section 3, we see that the lower convex curve $\mathcal{C}$ for $\alpha$ connects $s+2$ integer points:

$$
C_{0}=(0,1), C_{1}=(2,1), C_{2}=(5,2), \ldots, C_{s+1}=(p, q) \text { 局 }
$$

Here $C_{i}=\left(F_{2 i+1}, F_{2 i-1}\right)$ for $1 \leq i \leq s+1$. Let $\mathcal{C}^{\prime}$ be the convex curve connecting $C_{1}, \ldots, C_{s+1}$ (see Figure 11). Property (G2), for every $1 \leq i \leq s$, the segment $C_{i} C_{i+1}$ has exactly 2 integer points, $C_{i}$ and $C_{i+1}$. In other words, we have $\mathcal{C}^{\prime} \cap \mathbb{Z}^{2}=\left\{C_{1}, \ldots, C_{s+1}\right\}$.

Let $Q$ be the triangle defined in (5.1). By Lemma 4.1, an integer point $\mathbf{y}=\left(y_{2}, y_{1}\right) \in Q$ lies on $\mathcal{C}^{\prime}$ if and only if $P_{\mathbf{y}}$ is integer point free, where $P_{\mathbf{y}}$ was defined in (4.4) 6 In other words, we have:

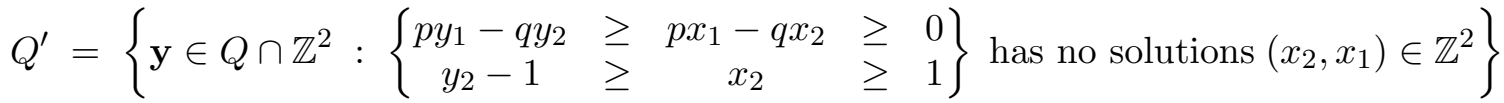

$$
\begin{aligned}
& =\mathcal{C}^{\prime} \cap \mathbb{Z}^{2} \text {. }
\end{aligned}
$$

The above is a PIP problem with parameters $\mathbf{y} \in Q$ and variables $\mathbf{x}=\left(x_{2}, x_{1}\right) \in \mathbb{Z}^{2}$. Note that the system has fixed length $m=4$. By Corollary 10.6, there exists a constant $d$, so that if $\left|\mathcal{C}^{\prime} \cap \mathbb{Z}^{2}\right|=s+1>d$ then there are 3 distinct points $\mathbf{y}_{1}, \mathbf{y}_{2}, \mathbf{y}_{3} \in \mathcal{C}^{\prime} \cap \mathbb{Z}^{2}$ with $\mathbf{y}_{3}=\left(\mathbf{y}_{1}+\mathbf{y}_{2}\right) / 2$. However, by the previous paragraph, the only integer points on $\mathcal{C}^{\prime}$ are $C_{1}, \ldots, C_{s+1}$, which are in convex position, see Property (G4). Thus, none among them can be the midpoint of two others. We get a contradiction. Therefore, (10.3) cannot hold in general.

Recall the PIP problem (10.6) with a 1-dimensional parameter $y^{\prime}$, i.e., $k=1$. From (10.3), we deduced $r \ll M$ in (10.8). This led to the observation that at least one interval $I^{\prime}$ must lie in a single piece $J_{i}$. The chain of deductions continued from there through Lemma 10.4 and Corollary 10.6 and led to the above contradiction. Therefore, we must have $r>M$, which implies $r \geq 2^{\varepsilon \phi}$ for some constant $\varepsilon=\varepsilon(m, n)>0$.

10.2. Implications. To summarize, Theorem 10.7 shows that a polynomial size decomposition into polyhedral pieces as in (10.2) does not exist. If one is willing to sacrifice the polyhedral structure of the pieces, then a polynomial size partition similar to (10.2) does in fact exist [ES08] (see also [Eis10]):

Theorem 10.8 (Eisenbrand and Shmonin). Fix $n$ and $k$. Let $A \mathbf{x} \leq \bar{b}$ be a PIP problem with a $k$-dimensional parameter space $W$. Then we can find in polynomial time a partition

$$
W=S_{1} \sqcup S_{2} \sqcup \ldots \sqcup S_{r},
$$

\footnotetext{
${ }^{5}$ Recall that the vertical coordinate is put in the first position.

${ }^{6}$ We take the first term in $\alpha$ to be 2 because of Remark 4.2
} 
where each $S_{i}$ is an integer projection of another polyhedron $S_{i}^{\prime} \subseteq \mathbb{R}^{m+\ell}$, defined as:

$$
S_{i}=\left\{\bar{b} \in \mathbb{R}^{m}: \exists \mathbf{t} \in \mathbb{Z}^{\ell}(\bar{b}, \mathbf{t}) \in S_{i}^{\prime}\right\} .
$$

Here $\ell=\ell(n)$ is a constant that depends only on $n$. All polyhedra $S_{i}^{\prime}$ can be found in polynomial time. The partition (10.15) satisfies all other properties as claimed in KPT.

Note that the integer projection of a polyhedron defined in the theorem is not necessarily a polyhedron as the following example shows.

Example 10.9. Consider the polytope $S^{\prime}=\left\{\left(y_{2}, y_{1}\right) \in \mathbb{R}^{2}: 0 \leq y_{2} \leq 1,0 \leq y_{1}-3 y_{2} \leq\right.$ $2\}$. The integer projection of $S^{\prime}$ on the coordinate $y_{1}$ is $S=[0,2] \cup[3,4]$ (see Figure 9).

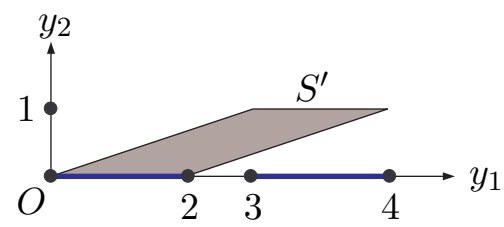

Figure 9. A polytope $S^{\prime}$ (shaded) and is integer projection (bold).

We emphasize that the proofs of Theorem 1.8 and Corollary 1.9 still hold if KPT is substituted by Theorem 10.8 (see [ES08]). Overall, the only discrepancy between KPT and Theorem 10.8 is about the structures of the pieces in the partition. This does not at all affect all known results about decision with 2 quantifiers or less. Worth mentioning is the polynomial time algorithm by Barvinok and Woods [BW03] on counting integer points in the integer projection of a polytope. This algorithm uses a weaker (valid) partitioning procedure also due to Kannan [Kan92, Lemma 3.1]. However, as we pointed out in Section 1.5, for 3 quantifiers or more, this structural discrepancy between KPT and Theorem 10.8 is of crucial importance.

\section{FinAl REMARKS AND OPEN PROBLEMS}

11.1. Niels Bohr, the inventor of quantum theory, is quoted saying:

"It is the hallmark of any deep truth that its negation is also a deep truth."

This roughly reflects our attitude towards KPT. A pioneer result at the time, it only slightly overstated the truth compared to the Eisenbrand-Shmonin theorem (Theorem 10.8). In fact, for many applications, including Kannan's Theorem 1.8 and Barvinok-Woods algorithm [BW03, Kannan's weaker result in [Kan92] is sufficient.

Let us emphasize that, of course, it would be natural to have a partition into convex (co-)polyhedra rather than general semilinear sets, since convex polyhedra are much easier to work with. The fact that it took nearly 30 years until KPT was disproved, shows both the delicacy and the technical difficulty of the issue. 
11.2. The gap in the proof of KPT (Theorem 3.1 in Kan90) could be traced to the following lines:

"... for each $(b, x) \in S_{i}$ (with $b \in P, x \in \mathbb{Z}^{n}$ ), there is a unique $y \in \mathbb{Z}^{\ell}$ so that $(b, x, y)$ belongs to $S_{i}^{\prime}$. In fact, each component of $y$ is of the form $F^{\prime}\lfloor F x\rfloor$, where $F^{\prime}, F$ are affine transformations. This is easily proved by induction on $n$, noting that (4.5) of [8], the $z$ is in fact forced to be $\lfloor\alpha+1-\beta\rfloor . "$

Here [8] refers to the conference proceedings version of paper [Kan92. In equation (4.5) of [Kan92, variable $z$ is in fact forced to be $\lfloor\alpha+1-\beta\rfloor$. However, the quantity $\alpha$ in (4.5) actually depends on $b$, which makes $\lfloor\alpha+1-\beta\rfloor$ a function of $b$ instead of a constant. This implies that $y$ in the above quoted paragraph could also depend on $b$. This technical error was perhaps due to the unclear notation $\alpha$, which does not reflect its dependence on $b$, or due to the complicated cross referencing between [Kan90] and Kan92].

11.3. There is a delicate difference between the treatment of (PIP) in Section 10.1 versus that in the integer programming literature (see e.g. CL98, V+07, VW08]). In the latter, the parameter space $W$ is also partitioned into convex polyhedra $P_{i}$, and over each $P_{i}$ the number of solutions $\mathbf{x}$ is given by a quasi-polynomial $p_{i}(\bar{b})$ in $\bar{b}$. However, since there are no test sets, this does not allow us to solve (PIP) for all $\bar{b}$. In other words, even though a quasi-polynomial $p_{i}(\bar{b})$ is obtained, which evaluates to $\left|K_{\bar{b}} \cap \mathbb{Z}^{n}\right|$, there is no easy way to test whether $p_{i}(\bar{b}) \neq 0$ for all $\bar{b}$ within $P_{i}$. In general, we prove in NP17b] that there are strong obstacles in using (short) generating functions to decide feasibility of Presburger sentences.

11.4. Now that we have Theorem 1.1, one can ask if the dimension 5 is tight. Observe that for three variables and three quantifiers, there is essentially a unique form of short Presburger sentence:

$$
\exists z \forall y \quad \exists x: \Phi(x, y, z) .
$$

Despite Theorem 1.10, KPT actually holds for a PIP problem $a x \leq f(y, z)$ with a single variable $x$, i.e., when $n=1$. Therefore, this sentence can be decided by the approach in $\left[\mathrm{NP} 17 \mathrm{a}\right.$. The only remaining special case of $\left(\mathrm{Short}_{-} \mathrm{PA}_{3}\right)$ is

$$
\exists z \forall y \exists \mathbf{x}: \Phi(\mathbf{x}, y, z), \quad \text { where } \mathbf{x} \in \mathbb{Z}^{2} .
$$

It would be interesting to see if this case is also NP-complete.

Similarly, for sentences (GIP), one can ask if dimension 6 in Theorem 1.3 can be lowered. We believe it can be, at least for the counting part (cf. [NP17c]).

11.5. Motivated in part by the Hilbert's tenth problem, Manders and Adleman [MA] (see also [GJ79, §A7.2]) proved the following classical result: feasibility over $\mathbb{N}$ of

$$
a x^{2}+b y=c
$$

is NP-complete, given $a, b, c \in \mathbb{Z}$. One can view our Theorem 1.2 as a related result, where a single quadratic equation and two linear inequalities $x, y \geq 0$ (over $\mathbb{Z}$ ) are replaced with a system of 24 linear inequalities. 
11.6. Minimizing polynomial functions over integer points in a convex polytope is an interesting problem of Integer Programming. Already for polynomials of degree 4 in two variables this is known to be NP-hard [DHKW06], but for lower degree polynomials some such problems can be solved in polynomial time [DHWZ16. The survey paper Kop12 contains extensive background on various related problems. Curiously, the following natural problem remains open:

Question 11.1. Let $n$ be fixed. Given a polytope $P \subset \mathbb{R}^{n}$ and a rational quadratic function $f: \mathbb{R}^{n} \rightarrow \mathbb{R}$, can the optimization problem $\min _{\mathbf{x} \in P \cap \mathbb{Z}^{n}} f(\mathbf{x})$ be solved in polynomial time?

The case $n=2$ was resolved positively in [DeW14]. Note that the case $n=3$ with $f$ homogeneous is known to have an FPTAS [HWZ17.

11.7. Our Theorem [1.7 strongly contrasts with the positive results in [DHK09], which require that all $f_{i}$ 's are linear. There, it is proved that optimizing over the Pareto minima can be done in polynomial time when $g$ is linear. Furthermore, if $g$ is non-linear then an FPTAS also exists. Here, we say that having even one $f_{i}$ quadratic is enough to make the problem hard.

Note that in Theorem [1.7 we use three polynomial functions, two or which are linear. It would be interesting to see if just two polynomial functions suffice for the hardness.

Acknowledgements. We are greatly indebted to Sasha Barvinok for many fruitful discussions and encouragement. We are also grateful to Iskander Aliev, Matthias Aschenbrenner, Artëm Chernikov, Fritz Eisenbrand, Lenny Fukshansky, Robert Hildebrand, Ravi Kannan, Oleg Karpenkov, Matthias Köppe, Rafi Ostrovsky and Kevin Woods for interesting conversations and helpful remarks. Special thanks to Jesús De Loera for suggesting hardness of Pareto optima as a possible application of our main results. This work was finished while both authors were in residence of the MSRI long term Combinatorics program in the Fall of 2017; we thank MSRI for the hospitality. The first author was partially supported by the UCLA Dissertation Year Fellowship. The second author was partially supported by the NSF. 


\section{REFERENCES}

[AB] S. Arora and B. Barak, Computational complexity. A modern approach, Cambridge Univ. Press, Cambridge, UK, 2009.

[Bar93] A. Barvinok, A polynomial time algorithm for counting integral points in polyhedra when the fimension is fixed, in Proc. 34th FOCS, IEEE, Los Alamitos, CA, 1993, 566-572.

[Bar06] A. Barvinok, The complexity of generating functions for integer points in polyhedra and beyond, in Proc. ICM, Vol. 3, EMS, Zürich, 2006, 763-787.

[Bar08] A. Barvinok, Integer points in polyhedra, EMS, Zürich, 2008.

[Bar17] A. Barvinok, Lattice points and lattice polytopes, to appear in Handbook of Discrete and Computational Geometry (third edition), CRC Press, Boca Raton, FL, 2017, 26 pp.

[BP99] A. Barvinok and J. E. Pommersheim, An algorithmic theory of lattice points in polyhedra, in New Perspectives in Algebraic Combinatorics, Cambridge Univ. Press, Cambridge, 1999, 91-147.

[BW03] A. Barvinok and K. Woods, Short rational generating functions for lattice point problems, Jour. AMS 16 (2003), 957-979.

[CL98] P. Clauss and V. Loechner, Parametric analysis of polyhedral iteration spaces, J. VLSI Signal Process. 19 (1998), 179-194.

[Coo72] D. C. Cooper, Theorem proving in arithmetic without multiplication, in Machine Intelligence (B. Meltzer and D. Michie, eds.), Edinburgh Univ. Press, 1972, 91-99.

[DHK09] J. A. De Loera, R. Hemmecke, M. Köppe, Pareto optima of multicriteria integer linear programs, INFORMS J. Comput. 21 (2009), 39-48.

[DHKW06] J. A. De Loera, R. Hemmecke, M. Köppe and R. Weismantel, Integer Polynomial Optimization in Fixed Dimension, Math. Oper. Research 31 (2006), 147-153.

[DeW14] A. Del Pia and R. Weismantel, Integer quadratic programming in the plane, in Proc. 25th SODA, ACM, New York, 2014, 840-846.

[DHWZ16] A. Del Pia, R. Hildebrand, R. Weismantel and K. Zemmer, Minimizing cubic and homogeneous polynomials over integers in the plane, Math. Oper. Res. 41 (2016), 511-530.

[Eis03] F. Eisenbrand, Fast integer programming in fixed dimension, in Proc. 11th ESA, Springer, Berlin, 2003, 196-207.

[Eis10] F. Eisenbrand, Integer programming and algorithmic geometry of numbers, in 50 years of Integer Programming, Springer, Berlin, 2010, 505-560.

[ES08] F. Eisenbrand and G. Shmonin, Parametric integer programming in fixed dimension, Math. Oper. Res. 33 (2008), 839-850.

[FR74] M. J. Fischer and M. O. Rabin, Super-Exponential Complexity of Presburger Arithmetic, in Proc. SIAM-AMS Symposium in Applied Mathematics, AMS, Providence, RI, 1974, 27-41.

[Für82] M. Fürer, The complexity of Presburger arithmetic with bounded quantifier alternation depth, Theoret. Comput. Sci. 18 (1982), 105-111.

[GJ79] M. R. Garey and D. S. Johnson, Computers and intractability. A guide to the theory of NPcompleteness, Freeman, San Francisco, CA, 1979.

[Grä87] E. Grädel, The complexity of subclasses of logical theories, Dissertation, Universität Basel, 1987.

[HWZ17] R. Hildebrand, R. Weismantel and K. Zemmer, An FPTAS for minimizing indefinite quadratic forms over integers in polyhedra, in Proc. 27th SODA, ACM, New York, 2016, 1715-1723.

[Kan90] R. Kannan, Test sets for integer programs, $\forall \exists$ sentences, in Polyhedral Combinatorics, AMS, Providence, RI, 1990, 39-47.

[Kan92] R. Kannan, Lattice translates of a polytope and the Frobenius problem, Combinatorica 12 (1992), 161-177.

[Kar13] O. Karpenkov, Geometry of continued fractions, Springer, Heidelberg, 2013.

[Khi64] A. Ya. Khinchin, Continued fractions, Univ. of Chicago Press, Chicago, IL, 1964.

[Kop12] M. Köppe, On the complexity of nonlinear mixed-integer optimization, Mixed integer nonlinear programming, 533-557, IMA Vol. Math. Appl., 154, Springer, New York, 2012.

[Lag85] J. Lagarias, The computational complexity of simultaneous Diophantine approximation problems, SIAM J. Comput. 14 (1985), 196-209.

[Len83] H. Lenstra, Integer programming with a fixed number of variables, Math. Oper. Res. 8 (1983), $538-548$.

[MA] K. Manders and L. Adleman, NP-complete decision problems for binary quadratics, J. Comput. System Sci. 16 (1978), 168-184.

[MM11] C. Moore and S. Mertens, The nature of computation, Oxford Univ. Press, Oxford, 2011. 
[NP17a] D. Nguyen and I. Pak, Complexity of short Presburger arithmetic, Proc. 49th STOC, ACM, 2017; arXiv:1704.00249

[NP17b] D. Nguyen and I. Pak, Complexity of short generating functions; $\operatorname{arXiv:1702.08660}$

[NP17c] D. Nguyen and I. Pak, The computational complexity of integer programming with alternations, Proc. 32nd CCC, 2017; arXiv:1702.08662.

[Opp78] D. C. Oppen, A $2^{2^{2^{p n}}}$ upper bound on the complexity of Presburger arithmetic, J. Comput. System Sci. 16 (1978), 323-332.

[Pap94] C. H. Papadimitriou, Computational complexity, Addison-Wesley, Reading, MA, 1994.

[Pre29] M. Presburger, Über die Vollständigkeit eines gewissen Systems der Arithmetik ganzer Zahlen, in welchem die Addition als einzige Operation hervortritt (in German), in Comptes Rendus du I congrès de Mathématiciens des Pays Slaves, Warszawa, 1929, 92-101.

[RL78] C. R. Reddy and D. W. Loveland, Presburger arithmetic with bounded quantifier alternation, in Proc. 10th STOC, ACM, 1978, 320-325.

[Sca84] B. Scarpellini, Complexity of subcases of Presburger arithmetic, Trans. AMS 284 (1984), 203218 .

[Sch86] A. Schrijver, Theory of linear and integer programming, John Wiley, Chichester, 1986.

[Sch97] U. Schöning, Complexity of Presburger arithmetic with fixed quantifier dimension, Theory Comput. Syst. 30 (1997), 423-428.

[SM73] L. J. Stockmeyer and A. R. Meyer, Word problems requiring exponential time: preliminary report, in Proc. Fifth STOC, ACM, New York, 1973, 1-9.

[V+07] S. Verdoolaege, R. Seghir, K. Beyls, V. Loechner and M. Bruynooghe, Counting integer points in parametric polytopes using Barvinok's rational functions, Algorithmica 48 (2007), 37-66.

[VW08] S. Verdoolaege and K. Woods, Counting with rational generating functions, J. Symbolic Comput. 43 (2008), 75-91.

[Wei97] V. D. Weispfenning, Complexity and uniformity of elimination in Presburger arithmetic, in Proc. 1997 ISSAC, ACM, New York, 1997, 48-53.

[Woo04] K. Woods, Rational Generating Functions and Lattice Point Sets, Ph.D. thesis, University of Michigan, 2004, 112 pp.

[Woo15] K. Woods, Presburger arithmetic, rational generating functions, and quasi-polynomials, J. Symb. Log. 80 (2015), 433-449.

[Zie95] G. Ziegler, Lectures on polytopes, Springer, New York, 1995. 